\title{
Structure Revision of Four Classes of Prenylated Aromatic Natural Products Based on a Rule for Diagnostic ${ }^{13} \mathrm{C}$ NMR Chemical Shifts
}

Fu-Cai Ren, ${ }^{\dagger, \S, l}$ Li-Xia Wang, ${ }^{\dagger, l}$ Yong-Feng Lv, ${ }^{\dagger,}$ Jiang-Miao Hu, ${ }^{\dagger, \$, *}$ Jun Zhou ${ }^{\dagger, \$, *}$

†State Key Laboratory of Phytochemistry and Plant Resources in West China, and Yunnan Key Laboratory of Natural Medicinal Chemistry, Kunming Institute of Botany, Chinese Academy of Sciences, Kunming 650201, China

${ }^{\ddagger}$ Key Laboratory of Pu-er Tea Science, Ministry of Education, and College of Food Science and Technology, Yunnan Agricultural University, Kunming 650201, China ${ }^{\S}$ University of Chinese Academy of Sciences, Beijing 100049, China

\section{Corresponding Author}

*E-mail: hujiangmiao@mail.kib.ac.cn (J.-M. Hu); jzhou@mail.kib.ac.cn (J. Zhou). 'F.-C. Ren and L.-X. Wang contributed equally to this work. 


\section{Table of Contents}

Table S1. Revisions of misassigned compounds bearing moiety A.

Table S2. Comparison of ${ }^{13} \mathrm{C}$ NMR data of epoxycannabigerolic acids and radstrictins

C and D. S3

Table S3. Revisions of misassigned compounds bearing moiety B. S4

Table S4. Revisions of misassigned compounds bearing moiety C. S5

Table S5. Revisions of misassigned compounds bearing moiety D. S6

Table S6. The reassigned NMR data of prenyl moiety of class B. S7

Table S7. The reassigned NMR data of prenyl moiety of class C ..S8

Table S8. The reassigned NMR data of prenyl moiety of class D. $\mathrm{S} 10$

NMR, HRMS, and IR Spectra for the Synthesized Compounds $\mathrm{S} 12$

References S44 
Table S1. Revisions of misassigned compounds bearing moiety A.

\begin{tabular}{|c|c|c|c|c|c|}
\hline No. & Name & Sources and references & Diagnostic carbon signals & $\begin{array}{l}\text { Revised } \\
\text { Class }\end{array}$ & $\begin{array}{l}\text { Revised Structure No. } \\
\text { and references }\end{array}$ \\
\hline $11 \mathrm{a}$ & Addisoniaflavanone I & Erythrina addisoniae ${ }^{1}$ & $69.8(\mathrm{~d}, \mathrm{C}-2), 78.1(\mathrm{~s}, \mathrm{C}-3)$ & $\mathrm{A} \rightarrow \mathrm{C}$ & $11^{2}$ \\
\hline $12 \mathrm{a}$ & Epoxynymphaeol C & Macaranga tanarius ${ }^{3}$ & $68.4(\mathrm{~d}, \mathrm{C}-2), 78.6(\mathrm{~s}, \mathrm{C}-3)$ & $\mathrm{A} \rightarrow \mathrm{C}$ & $12^{4}$ \\
\hline $14 a$ & Mortatarin A & Morus alba 6 & $66.8(\mathrm{~d}, \mathrm{C}-2), 78.7(\mathrm{~s}, \mathrm{C}-3)$ & $\mathrm{A} \rightarrow \mathrm{C}$ & $14^{7}$ \\
\hline $15 \mathbf{a}$ & Ammopiptanine A & Ammopiptanthus mongolicus $^{8}$ & $68.6(\mathrm{~d}, \mathrm{C}-2), 79.6(\mathrm{~s}, \mathrm{C}-3)$ & $\mathrm{A} \rightarrow \mathrm{C}$ & 15 (new) \\
\hline $18 \mathrm{a}$ & $\begin{array}{l}\text { 1,3,5-Trihydroxy-6-[2"', } 3^{\prime \prime \prime} \text {-epoxy-3"'-methyl- } \\
\text { butyl]-2-[2"-methyl-butanoyl] } \\
\text {-4-[3'-methyl-2'-butenyl]-benzene }\end{array}$ & Hypericum foliosum ${ }^{11}$ & $68.9(\mathrm{~d}, \mathrm{C}-2), 78.3(\mathrm{~s}, \mathrm{C}-3)$ & $\mathrm{A} \rightarrow \mathrm{C}$ & 18 (new) \\
\hline $19 a$ & Vismiaphenone $\mathrm{G}$ & Vismia cayennensis $^{12}$ & $69.6(\mathrm{~d}, \mathrm{C}-2), 78.5(\mathrm{~s}, \mathrm{C}-3)$ & $\mathrm{A} \rightarrow \mathrm{C}$ & 19 (new) \\
\hline 20a & 2',3'-Epoxy-homoedudiol & Erythrina melanacantha ${ }^{13}$ & $69.7(\mathrm{~d}, \mathrm{C}-2), 76.9(\mathrm{~s}, \mathrm{C}-3)$ & $\mathrm{A} \rightarrow \mathrm{C}$ & 20 (new) \\
\hline $25 \mathrm{a}$ & $\begin{array}{l}\text { 2'-[[3-Methyl-3-(4-methyl-3-penten-1-yl)-2- } \\
\text { oxiranyl]methyl]-3',5',6-trihydroxy-2-arylbenzofuran }\end{array}$ & Morus alba ${ }^{20}$ & $68.5(\mathrm{~d}, \mathrm{C}-2), 79.9(\mathrm{~s}, \mathrm{C}-3)$ & $\mathrm{A} \rightarrow \mathrm{C}$ & 25 (new) \\
\hline 26a & Epoxycannabigerol & Cannabis sativa $^{21}$ & $\begin{array}{l}66.9(\mathrm{~d}, \mathrm{C}-2), 78.9(\mathrm{~s}, \mathrm{C}-3) \\
67.2(\mathrm{~d}, \mathrm{C}-2), 81.9(\mathrm{~s}, \mathrm{C}-3)\end{array}$ & $\mathrm{A} \rightarrow \mathrm{C}$ & 26 (new) \\
\hline $27 \mathbf{a}$ & Epoxycannabigerolic acid & Cannabis sativa $^{21}$ & $67.3(\mathrm{~d}, \mathrm{C}-2), 81.4(\mathrm{~s}, \mathrm{C}-3)$ & $\mathrm{A} \rightarrow \mathrm{C}$ & 27 (new) \\
\hline
\end{tabular}


68.3 (d, C-2), 78.4 (s, C-3)

28a Hyperinakin

Hypericum nakamurai ${ }^{22}$

67.5 (d, C-2), 80.3 (s, C-3)

Erythrina fusca ${ }^{23}$

Teclea amanuensis ${ }^{25}$

89.9 (d, C-2), 71.7 (s, C-3) A $\rightarrow$ D

28 (new)

29a Fuscacarpan A

Vepris glomerate ${ }^{26}$

90.6 (d, C-2), 71.9 (s, C-3) A $\rightarrow$ D

$29^{24}$

30a Tecleamaniensine $B$

91.1 (d, C-2), 71.7 (s, C-3) A $\rightarrow$ D

30 (new)

$31^{27}$ 
Table S2. Comparison of ${ }^{13} \mathrm{C}$ NMR data of epoxycannabigerolic acids (27a) and radstrictins $\mathrm{C}$ and $\mathrm{D}$.

\begin{tabular}{|c|c|c|c|c|c|c|c|c|c|c|}
\hline \multirow[t]{2}{*}{ Comp. } & $\mathrm{C}-\mathbf{1}^{\prime}$ & $\mathrm{C}-2^{\prime}$ & C-3' & C-4' & C-5' & C-6' & C-1 & $\mathrm{C}-2$ & C-3 & C-4 \\
\hline & $\delta_{\mathrm{C}}(\mathrm{s})$ & $\delta_{\mathrm{C}}(\mathrm{s})$ & $\delta_{\mathrm{C}}(\mathrm{s})$ & $\delta_{\mathrm{C}}(\mathrm{s})$ & $\delta_{\mathrm{C}}(\mathrm{d})$ & $\delta_{\mathrm{C}}(\mathrm{s})$ & $\delta_{\mathrm{C}}(\mathrm{t})$ & $\delta_{\mathrm{C}}(\mathrm{d})$ & $\delta_{\mathrm{C}}(\mathrm{s})$ & $\delta_{\mathrm{C}}(\mathrm{q})$ \\
\hline 27a1 $(6,7$-trans $)$ & 106.1 & 151.4 & 116.1 & 138.8 & 107.0 & 156.0 & 26.7 & 66.9 & 78.9 & 18.1 \\
\hline $27 \mathbf{a} 2(6,7-c i s)$ & 105.0 & 153.0 & 110.9 & 145.1 & 108.4 & 153.8 & 26.0 & 67.2 & 81.9 & 17.7 \\
\hline radstrictin $\mathrm{C}$ & 105.1 & 163.2 & 103.9 & 142.1 & 111.8 & 157.5 & 26.0 & 69.1 & 77.7 & 22.1 \\
\hline radstrictin $\mathrm{D}$ & 104.7 & 151.5 & 115.9 & 141.6 & 107.7 & 155.0 & 26.1 & 69.0 & 77.3 & 21.8 \\
\hline \multicolumn{11}{|l|}{$\triangle(\mathbf{2 7} \mathbf{a}-$ radstrictin $\mathrm{C} / \mathrm{D})$} \\
\hline$\triangle(\mathbf{2 7} \mathbf{a} 2$ - radstrictin $C)$ & -0.1 & -10.2 & -7.0 & 3.0 & -3.4 & -3.7 & 0 & -1.9 & 4.2 & -4.4 \\
\hline$\triangle(\mathbf{2 7} \mathbf{a} 2$ - radstrictin $\mathrm{D})$ & 0.3 & 1.5 & 5.0 & 3.5 & 0.7 & -1.2 & -0.1 & -1.8 & 4.6 & -4.1 \\
\hline
\end{tabular}

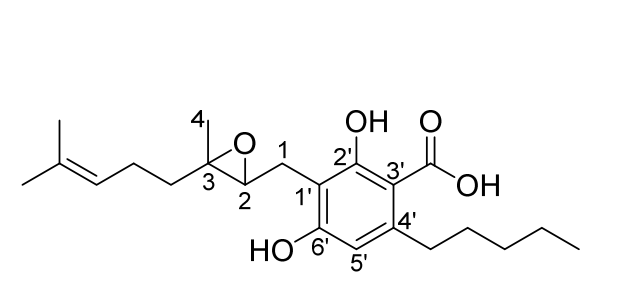

6,7-Epoxycannabigerolic acid

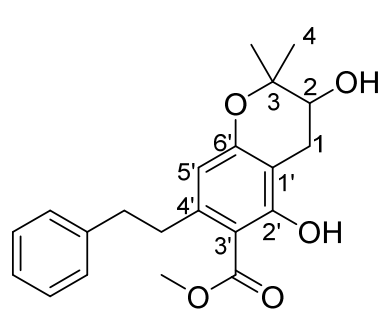

Radstrictin C

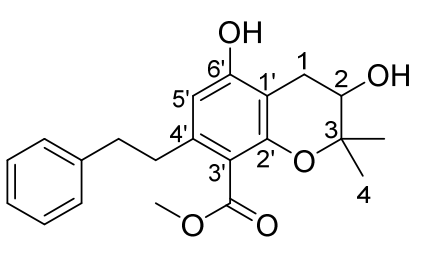

Radstrictin D 
Table S3. Revisions of misassigned compounds bearing moiety $B$.

\begin{tabular}{|c|c|c|c|c|c|}
\hline No. & Name & Sources and references & Diagnostic carbon signals & $\begin{array}{l}\text { Revised } \\
\text { Class }\end{array}$ & $\begin{array}{l}\text { Revised Structure No. } \\
\text { and references }\end{array}$ \\
\hline $32 \mathbf{a}$ & 3-(2,3-Dihydroxy-isopentyl)-4-hydroxy-acetophenone & Werneria ciliolate $^{28}$ & $69.8(\mathrm{~d}, \mathrm{C}-2), 79.1(\mathrm{~s}, \mathrm{C}-3)$ & $\mathrm{B} \rightarrow \mathrm{C}$ & $32^{29}$ \\
\hline $33 \mathbf{a}$ & Leptotic acid A & Euodia lepta ${ }^{30}$ & $69.9(\mathrm{~d}, \mathrm{C}-2), 79.1(\mathrm{~s}, \mathrm{C}-3)$ & $\mathrm{B} \rightarrow \mathrm{C}$ & 33 (no NMR report) \\
\hline $34 \mathbf{a}$ & Hostmaniane & Piper hostmannianum ${ }^{31}$ & $69.2(\mathrm{~d}, \mathrm{C}-2), 77.8(\mathrm{~s}, \mathrm{C}-3)$ & $\mathrm{B} \rightarrow \mathrm{C}$ & $9^{32}$ \\
\hline $35 \mathbf{a}$ & Moracin R & Morus mesozygia ${ }^{33}$ & $70.0(\mathrm{~d}, \mathrm{C}-2), 78.1(\mathrm{~s}, \mathrm{C}-3)$ & $\mathrm{B} \rightarrow \mathrm{C}$ & $\mathbf{3 5}^{34}$ \\
\hline $36 \mathbf{a}$ & Macasiamenene $\mathrm{R}$ & Macaranga siamensis ${ }^{35}$ & $70.1(\mathrm{~d}, \mathrm{C}-2), 76.1(\mathrm{~s}, \mathrm{C}-3)$ & $\mathrm{B} \rightarrow \mathrm{C}$ & $36^{35}$ \\
\hline $37 \mathbf{a}$ & Sanjoseolide & Dalea frutescens ${ }^{36}$ & $69.1(\mathrm{~d}, \mathrm{C}-2), 78.2(\mathrm{~s}, \mathrm{C}-3)$ & $\mathrm{B} \rightarrow \mathrm{C}$ & $37^{37}$ \\
\hline $38 \mathbf{a}$ & Gigasol & Angelica gigas ${ }^{38}$ & $68.8(\mathrm{~d}, \mathrm{C}-2), 79.6(\mathrm{~s}, \mathrm{C}-3)$ & $\mathrm{B} \rightarrow \mathrm{C}$ & 38 (new) \\
\hline $39 \mathbf{a}$ & Myricoidiol & Rapanea myricoides $^{39}$ & $67.9(\mathrm{~d}, \mathrm{C}-2), 79.7(\mathrm{~s}, \mathrm{C}-3)$ & $\mathrm{B} \rightarrow \mathrm{C}$ & $3^{40}$ \\
\hline $40 \mathrm{a}$ & $\begin{array}{l}\text { 1-(3-(2,3-Dihydroxy-3-methylbutyl)-4- } \\
\text { hydroxy-5-(3-methylbut-2-en-1-yl) } \\
\text { phenyl)ethan-1-one }\end{array}$ & Senecio gallicus ${ }^{41}$ & $90.2(\mathrm{~d}, \mathrm{C}-2), 71.9(\mathrm{~s}, \mathrm{C}-3)$ & $\mathrm{B} \rightarrow \mathrm{D}$ & $\mathbf{4 0}^{42}$ \\
\hline $41 \mathrm{a}$ & $\begin{array}{l}\text { 7-Hydroxy-5-methoxy-6-(2',3'-dihydroxy } \\
\text {-3'-methylbutyl)coumarin }\end{array}$ & Helicia nilagirica $^{43}$ & $91.8(\mathrm{~d}, \mathrm{C}-2), 71.7(\mathrm{~s}, \mathrm{C}-3)$ & $\mathrm{B} \rightarrow \mathrm{D}$ & $41^{44}$ \\
\hline $42 \mathbf{a}$ & $\begin{array}{l}\text { 7-Hydroxy-8-prenyl-6-(2',3'-dihydroxy } \\
\text {-3'-methylbutyl)coumarin }\end{array}$ & Helicia nilagirica $^{43}$ & $90.5(\mathrm{~d}, \mathrm{C}-2), 71.9(\mathrm{~s}, \mathrm{C}-3)$ & $\mathrm{B} \rightarrow \mathrm{D}$ & 42 (no NMR report) \\
\hline 43a & Dulcisxanthone G & Garcinia dulcis ${ }^{45}$ & $91.7(\mathrm{~d}, \mathrm{C}-2), 71.8(\mathrm{~d}, \mathrm{C}-3)$ & $\mathrm{B} \rightarrow \mathrm{D}$ & $43^{46}$ \\
\hline $44 a$ & Addisoniaflavanone II & Erythrina addisoniae $^{1}$ & $90.1(\mathrm{~d}, \mathrm{C}-2), 71.6(\mathrm{~s}, \mathrm{C}-3)$ & $\mathrm{B} \rightarrow \mathrm{D}$ & $44^{47}$ \\
\hline $45 \mathbf{a}$ & Aspernolide D & Aspergillus terreus ${ }^{48}$ & $89.1(\mathrm{~d}, \mathrm{C}-2), 72.4(\mathrm{~s}, \mathrm{C}-3)$ & $\mathrm{B} \rightarrow \mathrm{D}$ & $\mathbf{4 5}^{49}$ \\
\hline $46 \mathbf{a}$ & Floribol C & Grevillea floribunda $a^{50}$ & $90.9(\mathrm{~d}, \mathrm{C}-2), 72.7(\mathrm{~s}, \mathrm{C}-3)$ & $\mathrm{B} \rightarrow \mathrm{D}$ & 46 (new) \\
\hline
\end{tabular}


Table S4. Revisions of misassigned compounds bearing moiety $\mathrm{C}$.

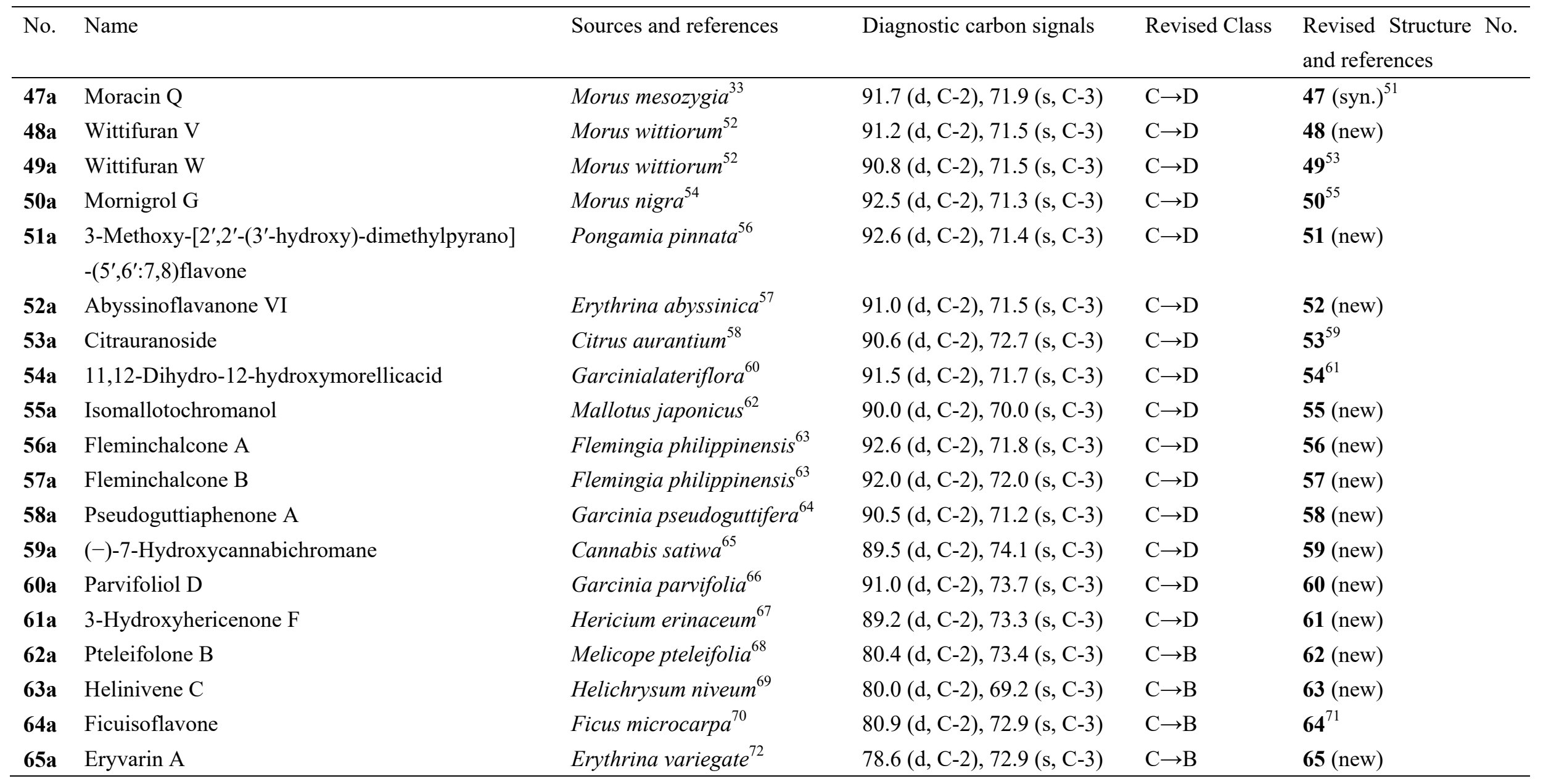


Table S5. Revisions of misassigned compounds bearing moiety D.

\begin{tabular}{|c|c|c|c|c|c|}
\hline No. & Name & Sources and references & Diagnostic carbon signals & Revised Class & Revised Structure No. and references \\
\hline $66 a$ & Sigmotriol & Erythrina sigmoidea ${ }^{73}$ & 70.5 (d, C-2), 78.7 (s, C-3) & $\mathrm{D} \rightarrow \mathrm{C}$ & $66^{2}$ \\
\hline $67 a$ & Sterenin J & Stereum hirsutum ${ }^{74}$ & $69.4(\mathrm{~d}, \mathrm{C}-2), 79.1(\mathrm{~s}, \mathrm{C}-3)$ & $\mathrm{D} \rightarrow \mathrm{C}$ & 67 (new) \\
\hline
\end{tabular}


Table S6. The reassigned NMR data of prenyl moiety of class B.

\begin{tabular}{llllll}
\hline Comp. no & $\mathrm{C}-1$ & $\mathrm{C}-2$ & $\mathrm{C}-3$ & $\mathrm{C}-4$ & $\mathrm{C}-5$ \\
\cline { 2 - 6 } & $\delta_{\mathrm{C}}(\mathrm{t})$ & $\delta_{\mathrm{C}}(\mathrm{d})$ & $\delta_{\mathrm{C}}(\mathrm{s})$ & $\delta_{\mathrm{C}}(\mathrm{q})$ & $\delta_{\mathrm{C}}(\mathrm{q}$ or $\mathrm{t})$ \\
\hline $\mathbf{6 2}$ & 25.4 & 80.4 & 73.4 & 22.7 & 26.4 \\
$\mathbf{6 3}$ & 27.1 & 80.0 & 69.2 & 20.2 & 20.3 \\
$\mathbf{6 4}$ & 34.5 & 80.9 & 72.9 & 25.4 & 25.5 \\
$\mathbf{6 5}$ & 27.0 & 78.6 & 72.9 & 25.0 & 26.1 \\
\hline
\end{tabular}


Table S7. The reassigned NMR data of prenyl moiety of class C.

\begin{tabular}{llllll}
\hline \multirow{2}{*}{ Comp. no } & $\mathbf{C - 1}$ & $\mathbf{C - 2}$ & $\mathbf{C - 3}$ & $\mathbf{C}-\mathbf{4}$ & $\mathbf{C - 5}$ \\
\cline { 2 - 6 } & $\delta_{\mathrm{C}}(\mathrm{t})$ & $\delta_{\mathrm{C}}(\mathrm{d})$ & $\delta_{\mathrm{C}}(\mathrm{s})$ & $\delta_{\mathrm{C}}(\mathrm{q})$ & $\delta_{\mathrm{C}}(\mathrm{q}$ or $\mathrm{t})$ \\
\hline $\mathbf{1 1}$ & 32.3 & 69.8 & 78.1 & 20.7 & 26.2 \\
$\mathbf{1 2}$ & 25.0 & 68.4 & 78.6 & 22.2 & 24.8 \\
$\mathbf{1 3}$ & 25.4 & 68.4 & 79.8 & 21.1 & 25.4 \\
$\mathbf{1 4}$ & 24.7 & 66.8 & 78.7 & 20.8 & 25.1 \\
$\mathbf{1 5}$ & 25.6 & 68.6 & 79.6 & 21.2 & 25.8 \\
$\mathbf{1 6}$ & 29.6 & 69.8 & 76.4 & 21.8 & 24.4 \\
$\mathbf{1 7}$ & 29.9 & 70.6 & 77.1 & 22.3 & 24.9 \\
$\mathbf{1 8}$ & 26.2 & 68.9 & 78.3 & 22.1 & 24.9 \\
$\mathbf{1 9}$ & 26.9 & 69.6 & 78.5 & 20.5 & 25.3 \\
$\mathbf{2 0}$ & 31.1 & 69.7 & 76.9 & 22.2 & 24.8 \\
$\mathbf{2 1}$ & 29.9 & 70.7 & 76.6 & 19.9 & 25.4 \\
$\mathbf{2 2}$ & 32.8 & 70.4 & 78.5 & 21.1 & 26.6 \\
$\mathbf{2 3}$ & 28.4 & 69.9 & 76.3 & 21.9 & 24.6 \\
$\mathbf{2 4}$ & 32.1 & 70.5 & 77.9 & 21.2 & 25.8 \\
$\mathbf{2 5}$ & 27.9 & 68.5 & 79.9 & 18.7 & 39.3 \\
$\mathbf{2 6}$ & 26.0 & 68.3 & 78.4 & 17.8 & 36.9 \\
& 26.0 & 67.3 & 81.4 & 17.8 & 36.9 \\
$\mathbf{2 7}$ & 26.7 & 66.9 & 78.9 & 18.1 & 38.8 \\
& 26.0 & 67.2 & 81.9 & 17.7 & 37.1 \\
$\mathbf{2 8}$ & 25.3 & 67.5 & 80.3 & 19.2 & 37.3 \\
$\mathbf{3 2}$ & 31.8 & 69.8 & 79.1 & 21.2 & 26.5 \\
\hline
\end{tabular}




\begin{tabular}{llllll}
\hline $\mathbf{3 3}$ & 31.9 & 69.9 & 79.1 & 21.5 & 25.8 \\
$\mathbf{9}$ & 31.1 & 69.2 & 77.8 & 22.0 & 25.0 \\
$\mathbf{3 5}$ & 32.4 & 70.0 & 78.1 & 20.7 & 26.2 \\
$\mathbf{3 6}$ & 30.5 & 70.1 & 76.1 & 21.9 & 24.7 \\
$\mathbf{3 7}$ & 25.9 & 69.1 & 78.2 & 22.6 & 24.6 \\
$\mathbf{3 8}$ & 31.8 & 68.8 & 79.6 & 21.1 & 26.5 \\
$\mathbf{3 9}$ & 31.1 & 67.9 & 79.7 & 19.0 & 37.6 \\
$\mathbf{6 6}$ & 32.2 & 70.5 & 78.7 & 21.1 & 25.7 \\
$\mathbf{6 7}$ & 27.7 & 69.4 & 79.1 & 20.9 & 25.5 \\
\hline
\end{tabular}


Table S8. The reassigned NMR data of prenyl moiety of class D.

\begin{tabular}{llllll}
\hline Comp. no & $\mathbf{C - 1}$ & $\mathbf{C - 2}$ & $\mathbf{C - 3}$ & $\mathbf{C - 4}$ & $\mathbf{C - 5}$ \\
\cline { 2 - 6 } & $\delta_{\mathrm{C}}(\mathrm{t})$ & $\delta_{\mathrm{C}}(\mathrm{d})$ & $\delta_{\mathrm{C}}(\mathrm{s})$ & $\delta_{\mathrm{C}}(\mathrm{q})$ & $\delta_{\mathrm{C}}(\mathrm{q}$ or $\mathrm{t})$ \\
\hline $\mathbf{2 9}$ & 27.7 & 89.9 & 71.7 & 23.8 & 25.5 \\
$\mathbf{3 0}$ & 33.7 & 90.6 & 71.9 & 24.1 & 26.2 \\
$\mathbf{3 1}$ & 30.6 & 91.1 & 71.7 & 24.2 & 26.1 \\
$\mathbf{4 0}$ & 30.4 & 90.2 & 71.9 & 24.2 & 25.7 \\
$\mathbf{4 1}$ & 27.9 & 91.8 & 71.7 & 24.1 & 26.0 \\
$\mathbf{4 2}$ & 29.1 & 90.5 & 71.9 & 24.3 & 25.7 \\
$\mathbf{4 3}$ & 26.8 & 91.7 & 71.8 & 24.1 & 25.3 \\
$\mathbf{4 4}$ & 31.2 & 90.1 & 71.6 & 25.3 & 26.1 \\
$\mathbf{4 5}$ & 30.5 & 89.1 & 72.4 & 23.9 & 25.8 \\
$\mathbf{4 6}$ & 30.0 & 90.9 & 72.7 & 25.2 & 25.6 \\
$\mathbf{4 7}$ & 31.1 & 91.7 & 71.9 & 26.0 & 26.5 \\
$\mathbf{4 8}$ & 30.6 & 91.2 & 71.5 & 25.4 & 26.0 \\
$\mathbf{4 9}$ & 28.5 & 90.8 & 71.5 & 25.5 & 26.1 \\
$\mathbf{5 0}$ & 25.5 & 92.5 & 71.3 & 25.5 & 25.8 \\
$\mathbf{5 1}$ & 27.9 & 92.6 & 71.4 & 25.5 & 26.0 \\
$\mathbf{5 2}$ & 30.6 & 91.0 & 71.5 & 23.1 & 26.8 \\
$\mathbf{5 3}$ & 30.8 & 90.6 & 72.7 & 26.5 & 25.3 \\
$\mathbf{5 4}$ & 26.9 & 91.5 & 71.7 & 24.3 & 28.8 \\
$\mathbf{5 5}$ & 26.7 & 90.0 & 70.0 & 22.9 & 26.3 \\
$\mathbf{5 6}$ & 28.1 & 92.6 & 71.8 & 26.3 & 26.4 \\
$\mathbf{5 7}$ & 29.8 & 92.0 & 72.0 & 25.5 & 26.5 \\
\hline & & & & & \\
\hline
\end{tabular}




\begin{tabular}{llllll}
\hline $\mathbf{5 8}$ & 29.3 & 90.5 & 71.2 & 23.7 & 25.6 \\
$\mathbf{5 9}$ & 27.5 & 89.5 & 74.1 & 23.1 & 37.1 \\
$\mathbf{6 0}$ & 26.7 & 91.0 & 73.7 & 22.7 & 36.7 \\
$\mathbf{6 1}$ & 27.0 & 89.2 & 73.3 & 24.7 & 49.2 \\
\hline
\end{tabular}


NMR, HRMS, and IR Spectra for the Synthesized Compounds

${ }^{1} \mathrm{H}$ NMR spectrum $\left(400 \mathrm{MHz}, \mathrm{CDCl}_{3}\right)$ of compound $\mathbf{1}$.

\begin{tabular}{|c|c|}
\hline 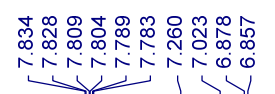 & 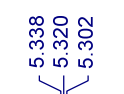 \\
\hline
\end{tabular}
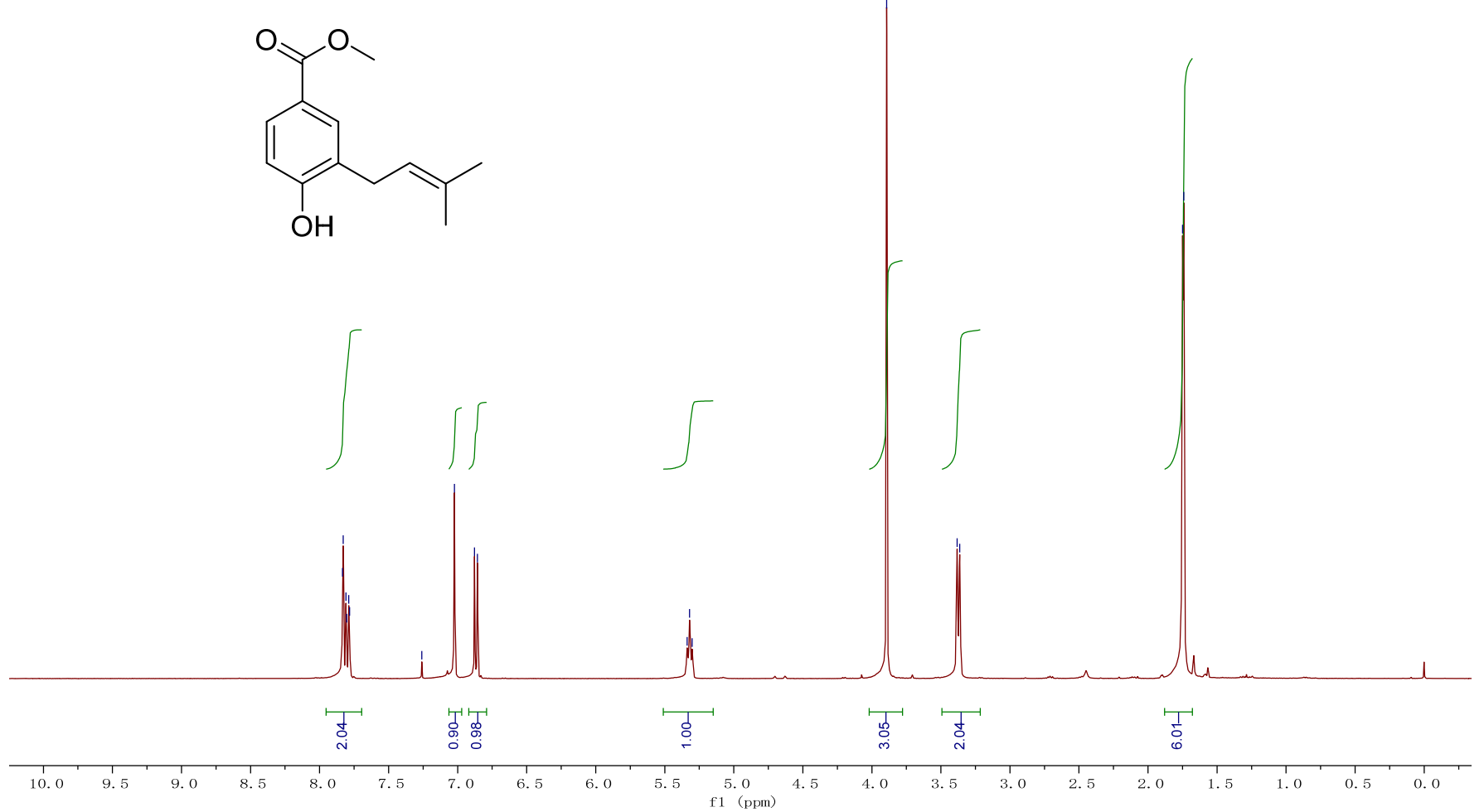
${ }^{13} \mathrm{C}$ and DEPT NMR spectra (100 MHz, $\left.\mathrm{CDCl}_{3}\right)$ of compound $\mathbf{1}$.
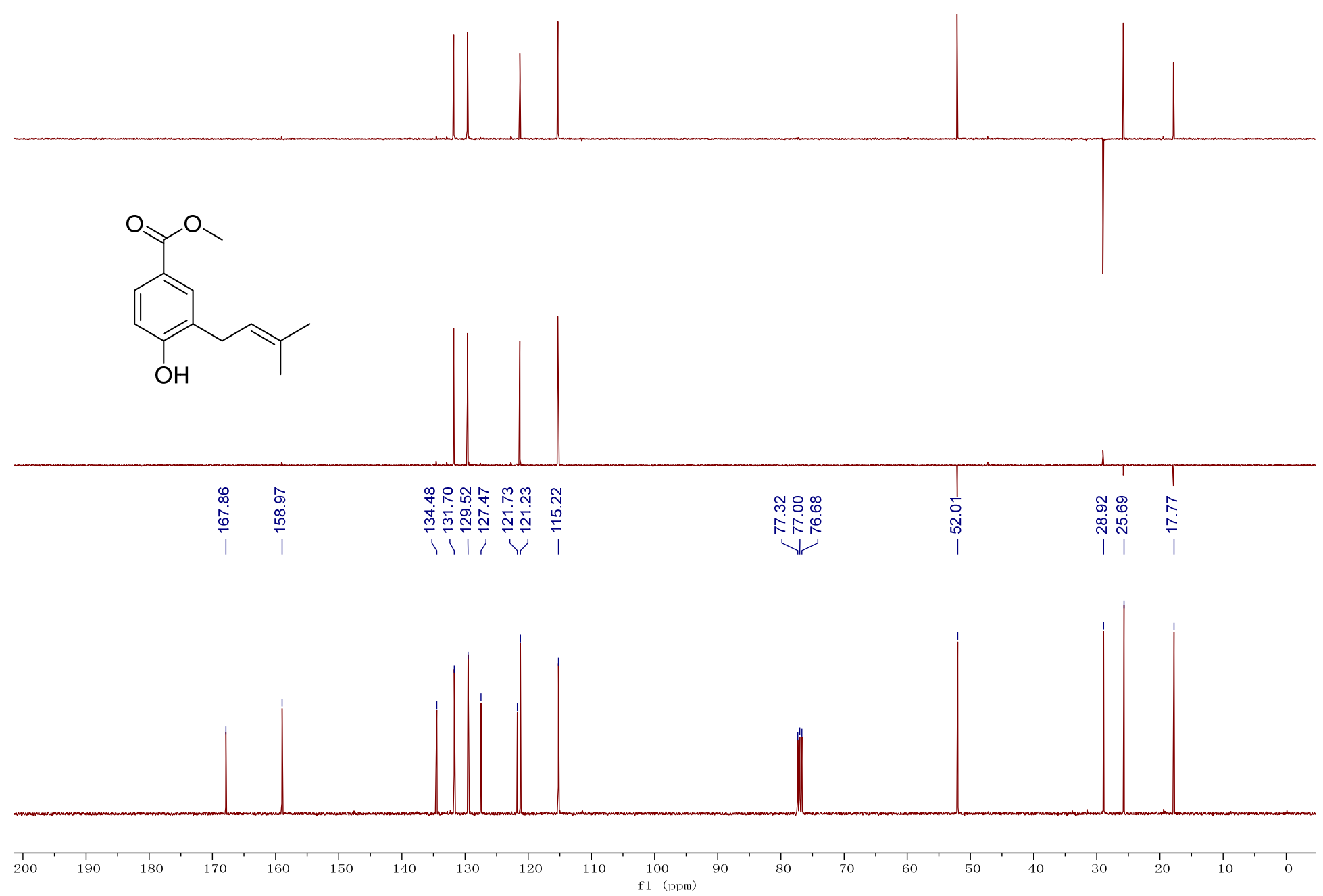
${ }^{1} \mathrm{H}$ NMR spectrum $\left(400 \mathrm{MHz}, \mathrm{CDCl}_{3}\right)$ of compound 2.

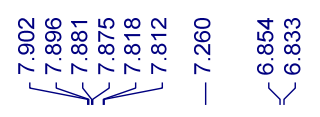

㩆絽

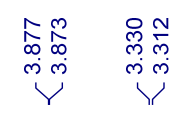

$\stackrel{\substack{i \\ i}}{i}$

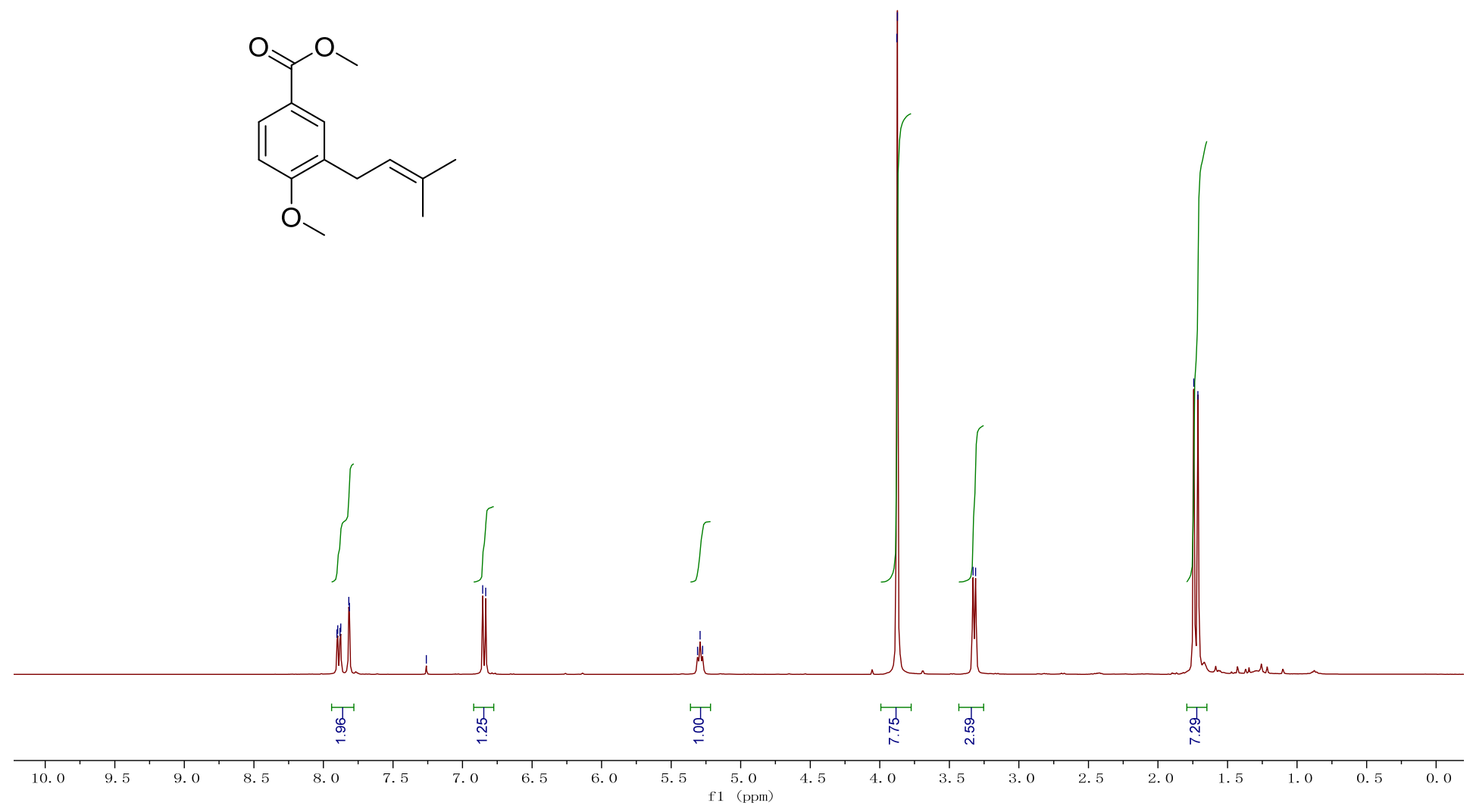

S14 
${ }^{13} \mathrm{C}$ and DEPT NMR spectra (100 MHz, $\left.\mathrm{CDCl}_{3}\right)$ of compound 2.

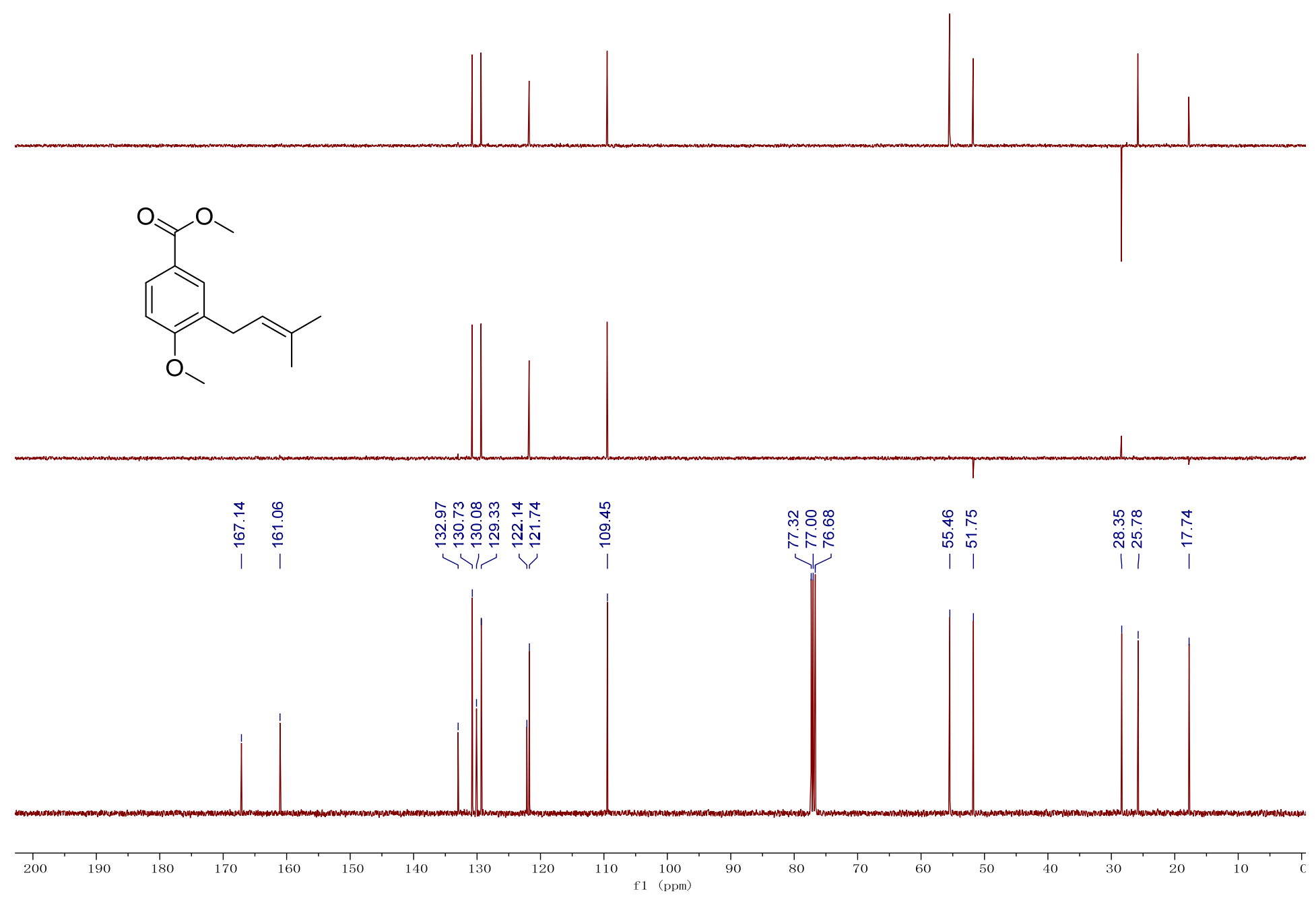


HREIMS spectrum of compound 2.

\section{Elemental Composition Report}

Page 1

Single Mass Analysis (displaying only valid results)

Tolerance $=10.0$ PPM / DBE: $\min =-1.5, \max =50.0$

Selected filters: None

Monoisotopic Mass, Odd and Even Electron lons

9 formula(e) evaluated with 1 results within limits (up to 50 closest results for each mass)

$\begin{array}{lll}\text { C: } 0-200 & \text { H: } 0-400 & \text { O: } 2-4\end{array}$

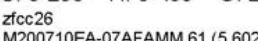

234.1259

Autospec
Vottage $1+$
23.3

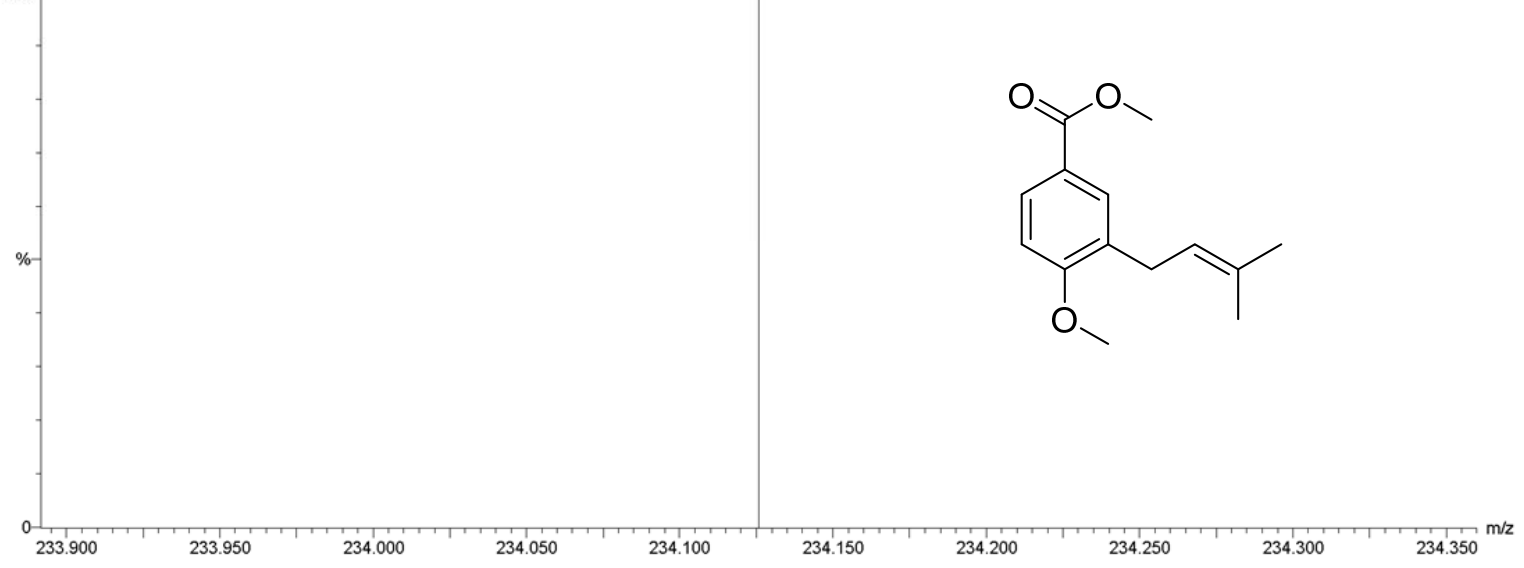

$\begin{array}{lll}\text { Minimum: } & 500.0 \quad 10.0 & -1.5 \\ \text { Maximum: } & 50.0\end{array}$

Mass Calc. Mass mDa PPM DBE i-FIT Formula

$\begin{array}{lllllllll}234.1259 & 234.1256 & 0.3 & 1.3 & 6.0 & 5546027.5 & \text { C14 } & \text { H18 } & 03\end{array}$ 
IR spectrum of compound 2 .

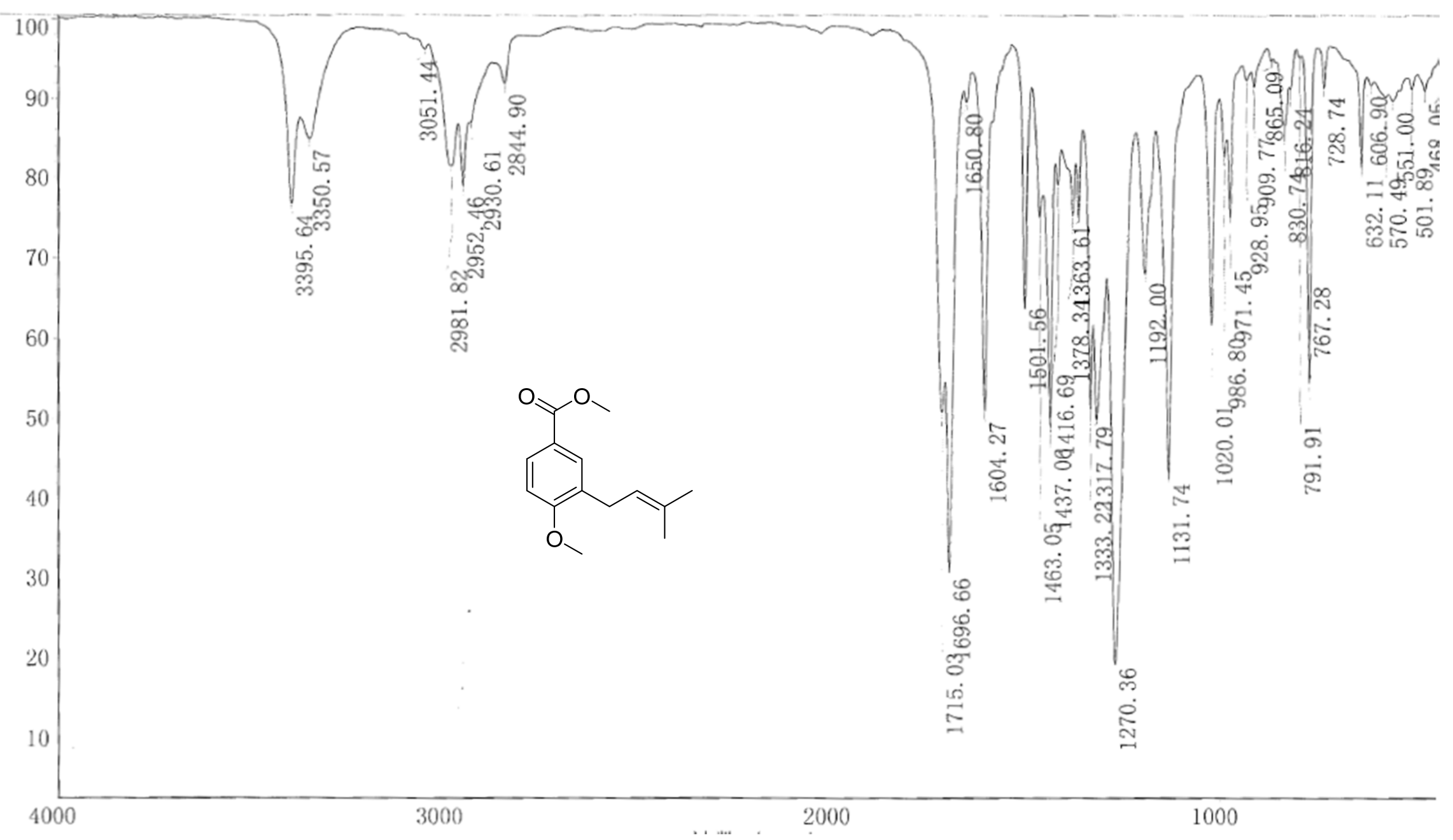


${ }^{1} \mathrm{H}$ NMR spectrum $\left(400 \mathrm{MHz}, \mathrm{CDCl}_{3}\right)$ of compound 3.

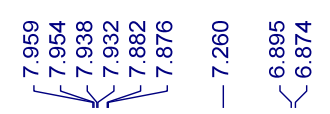

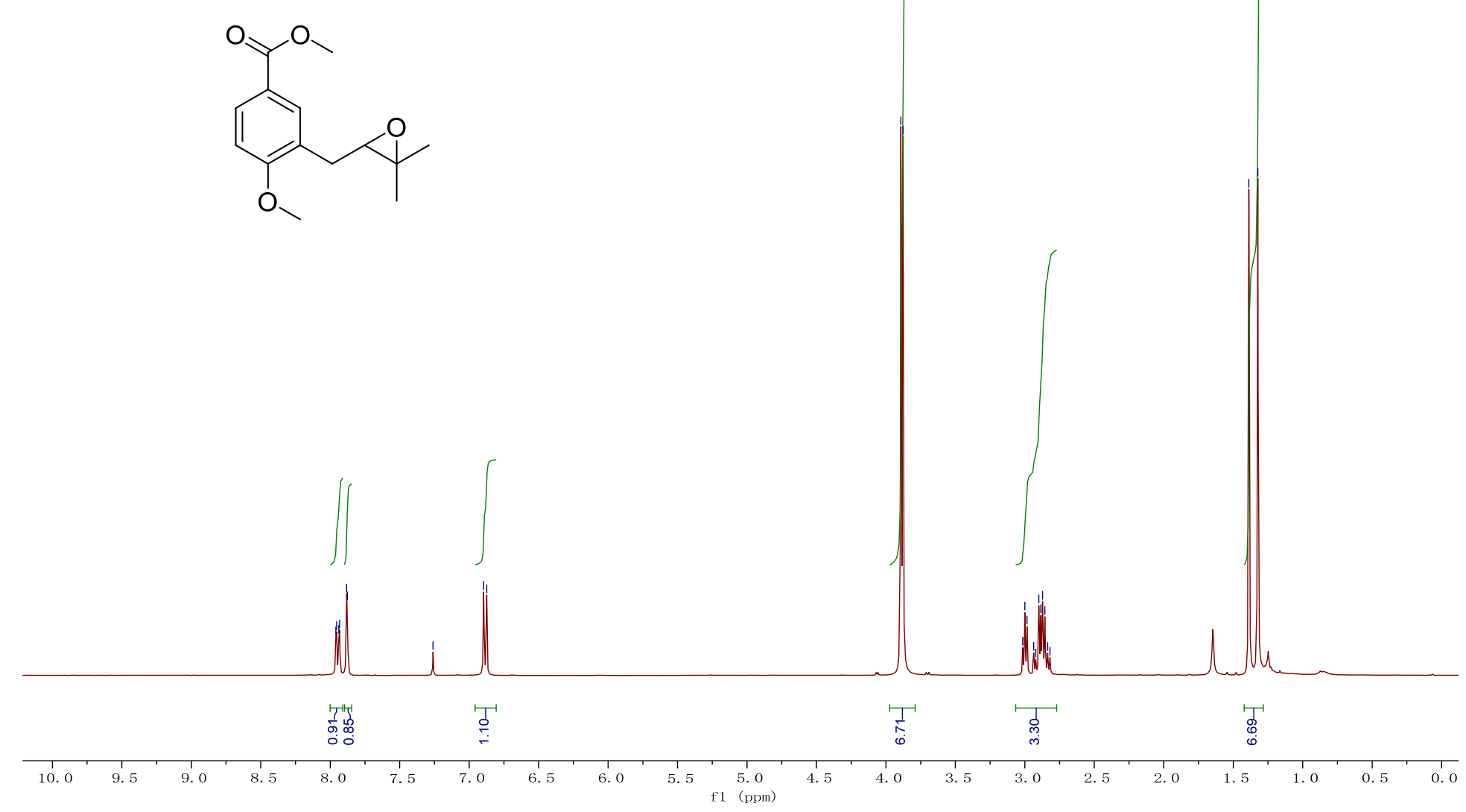

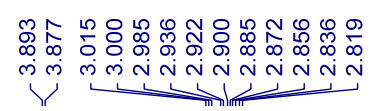

恖政

S18 
${ }^{13} \mathrm{C}$ and DEPT NMR spectra $\left(100 \mathrm{MHz}, \mathrm{CDCl}_{3}\right)$ of compound 3.
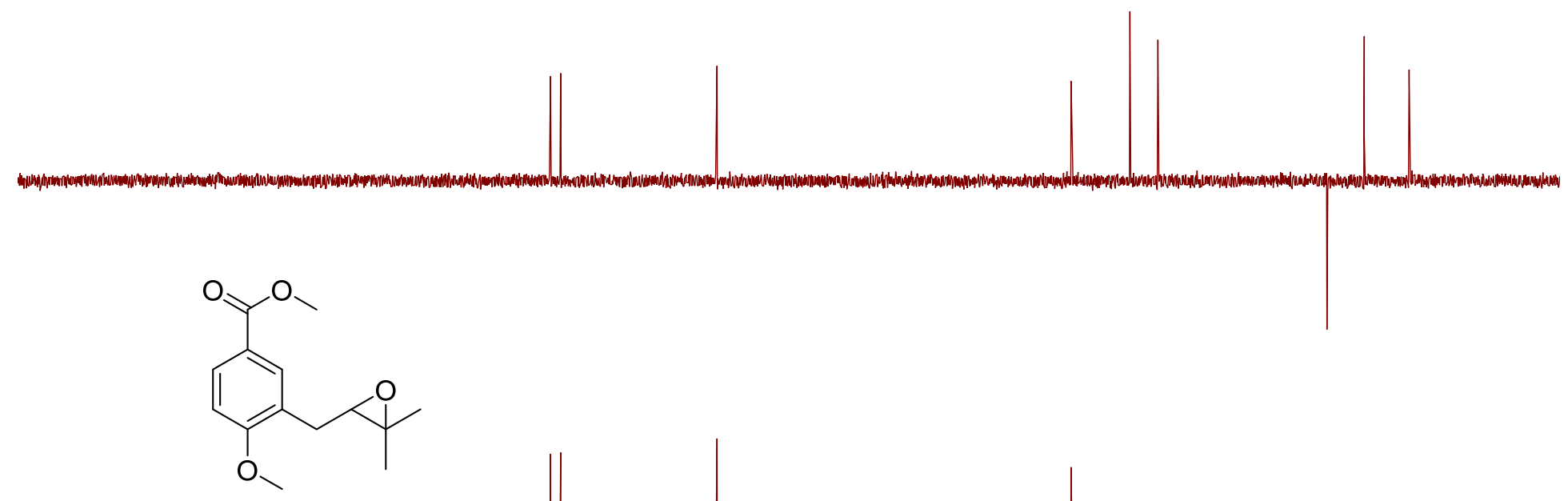

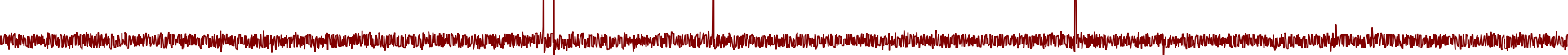

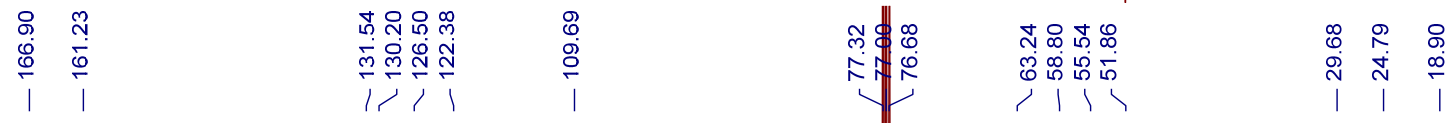

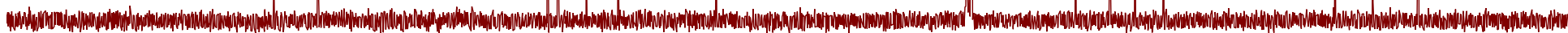

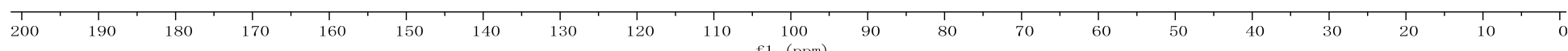


HRESIMS spectrum of compound 3 .
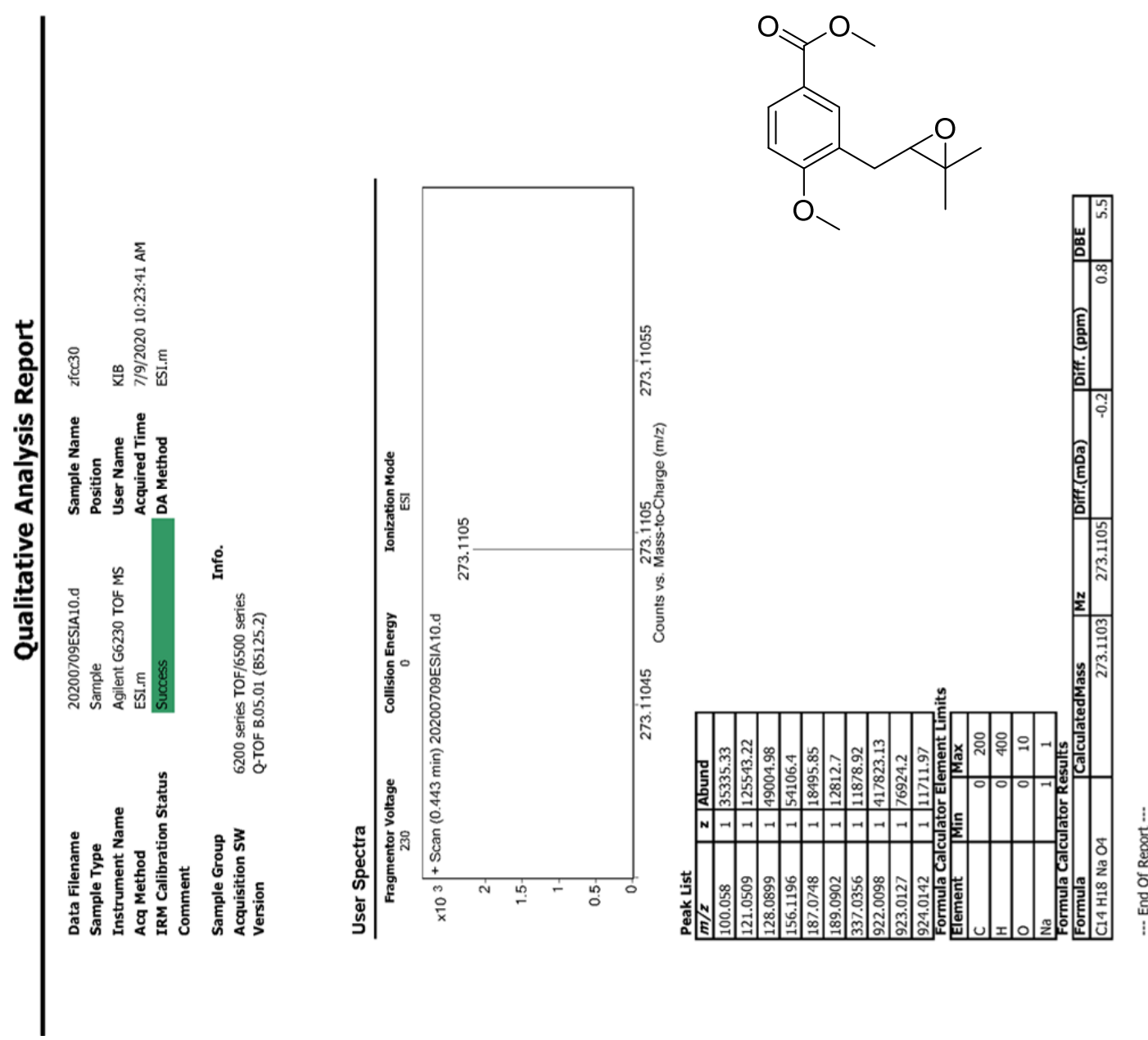
IR spectrum of compound $\mathbf{3}$.

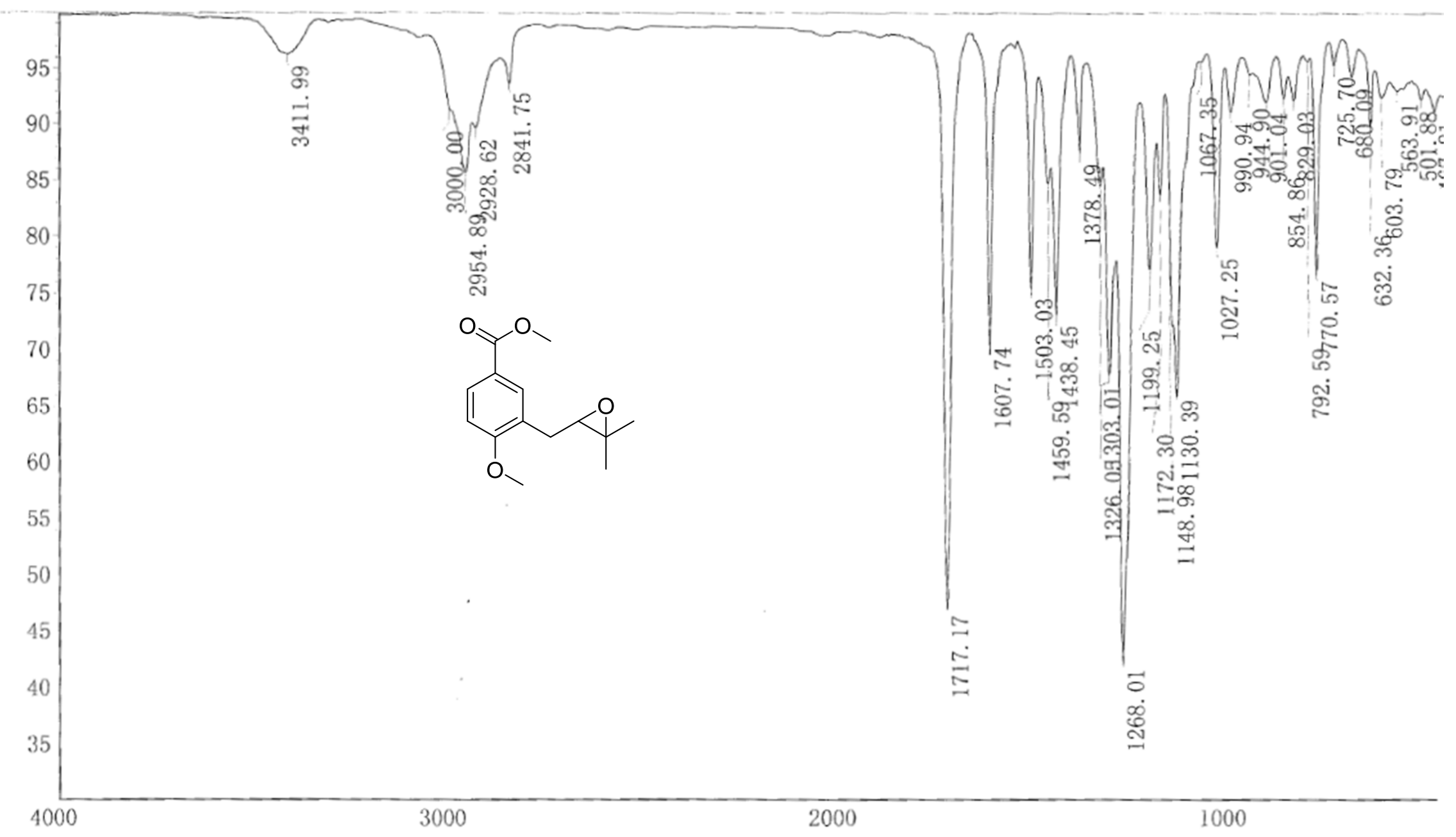


${ }^{1} \mathrm{H}$ NMR spectrum (400 MHz, DMSO- $d_{6}$ ) of compound 4.
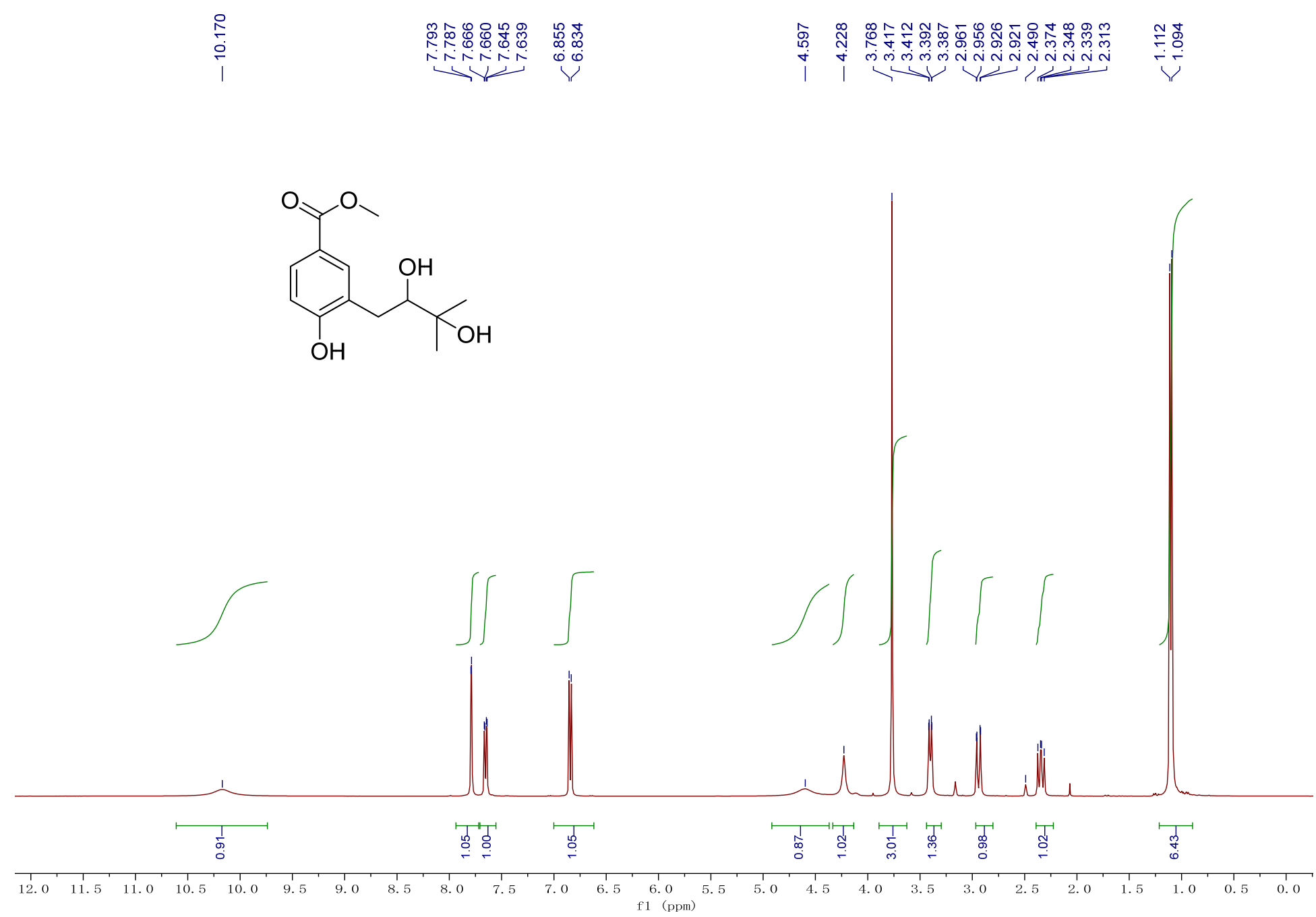
${ }^{13} \mathrm{C}$ and DEPT NMR spectra (100 MHz, DMSO- $\left.d_{6}\right)$ of compound 4.
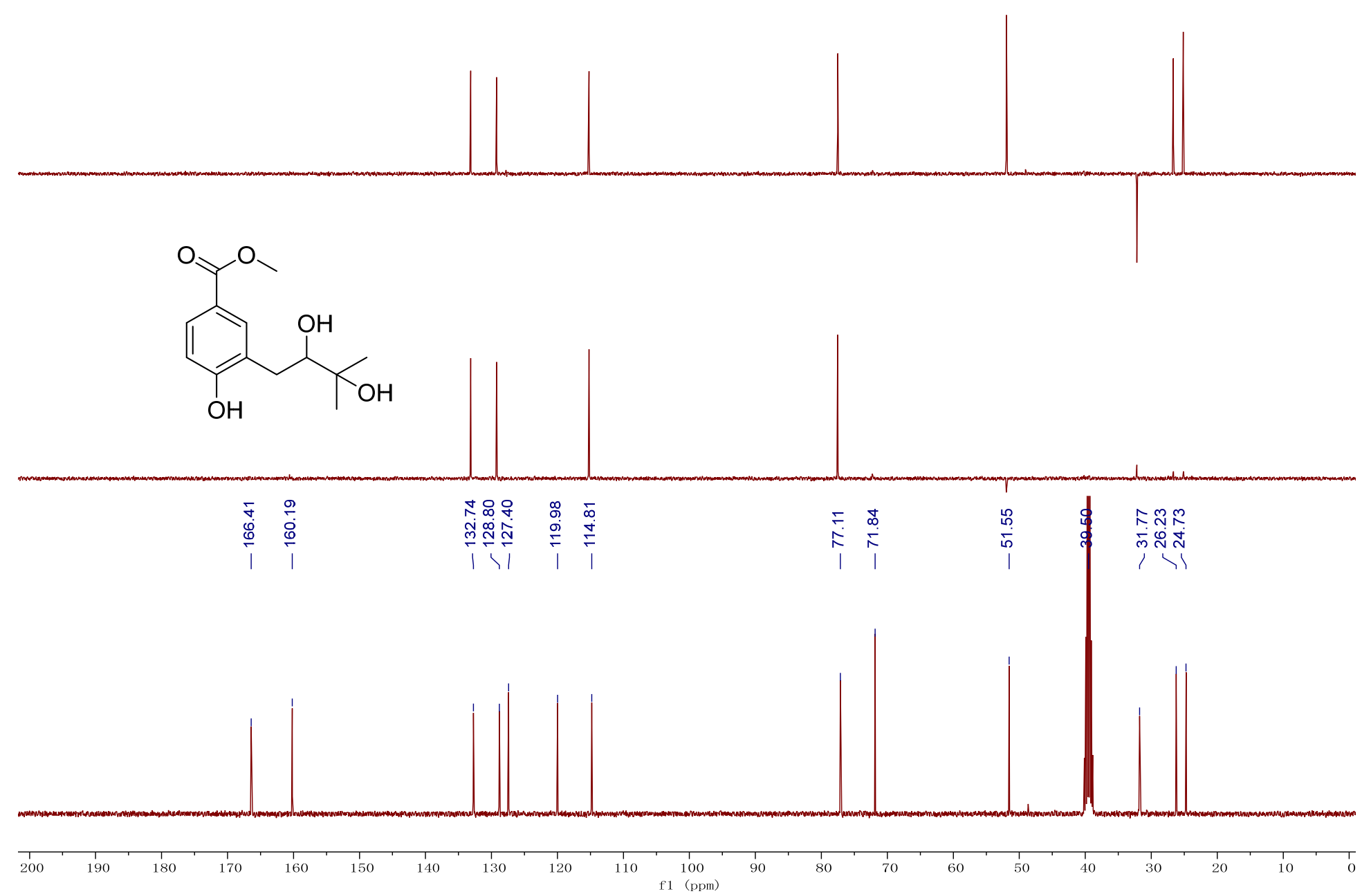
${ }^{1} \mathrm{H}$ NMR spectrum $\left(400 \mathrm{MHz}, \mathrm{CDCl}_{3}\right)$ of compound 5.

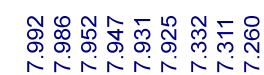

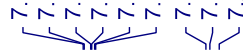

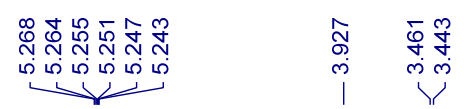

交
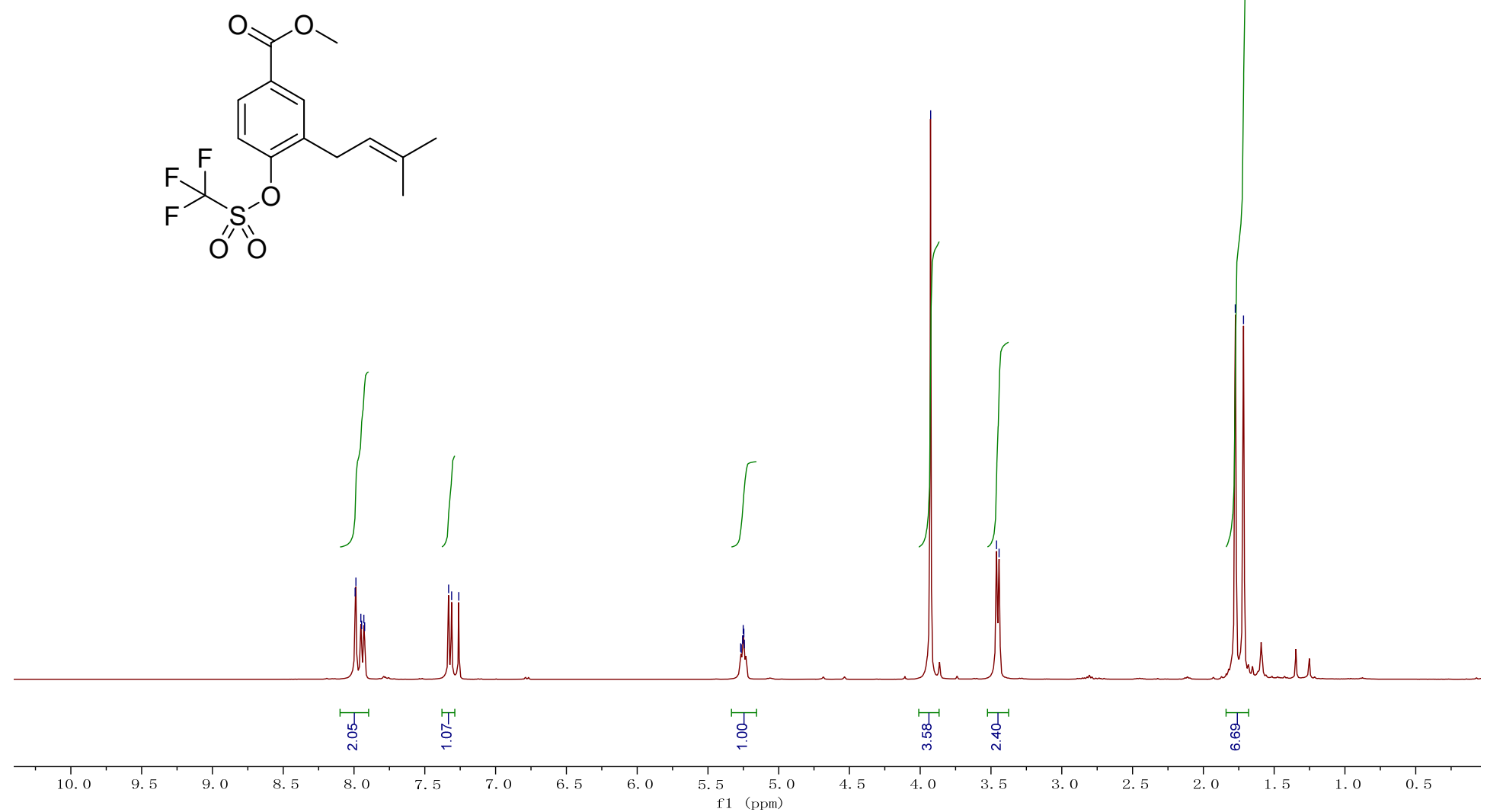
${ }^{13} \mathrm{C}$ and DEPT NMR spectra $\left(100 \mathrm{MHz}, \mathrm{CDCl}_{3}\right)$ of compound $\mathbf{5}$.
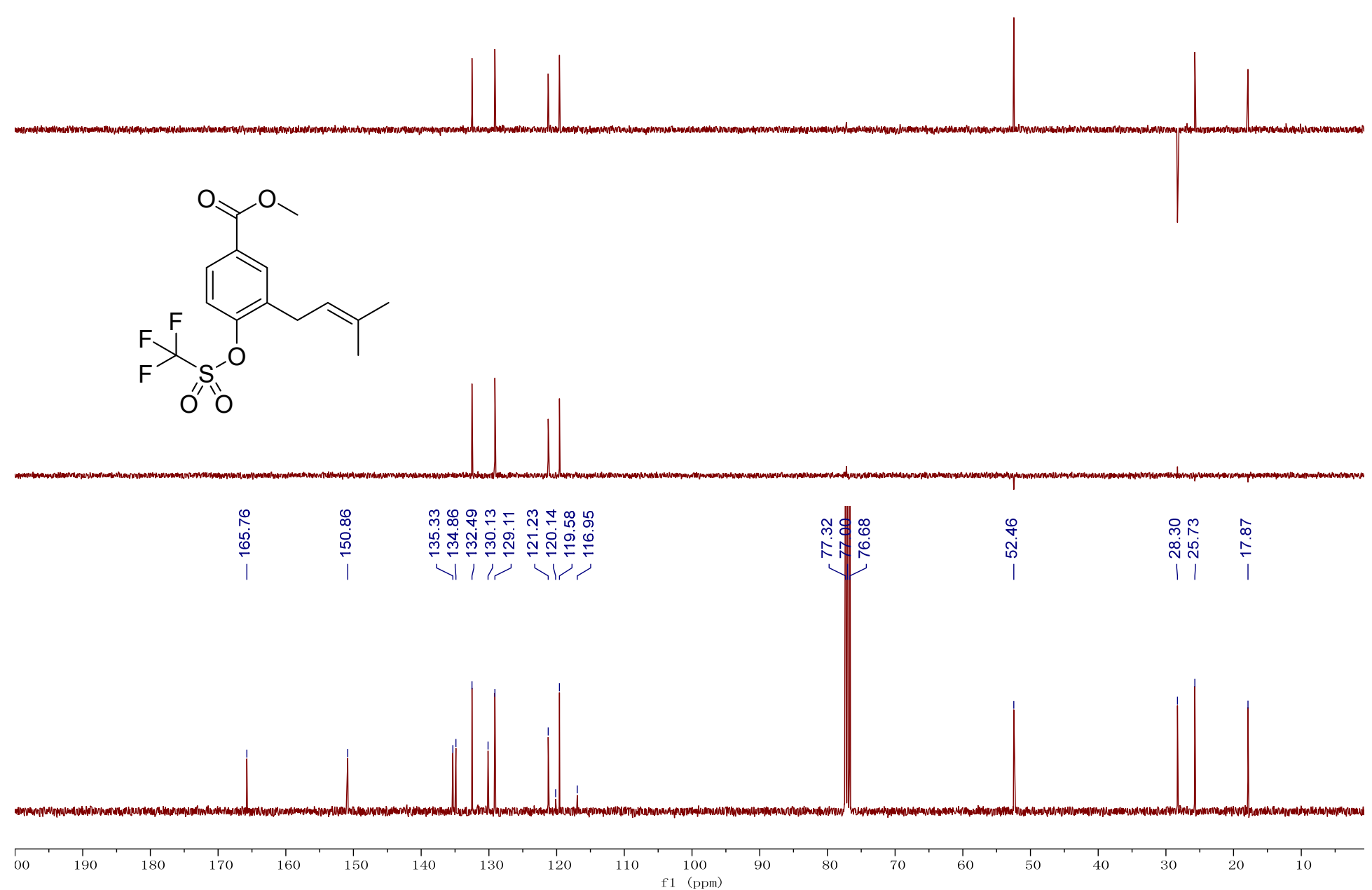
HREIMS spectrum of compound $\mathbf{5}$.

\section{Elemental Composition Report}

Page 1

Single Mass Analysis (displaying only valid results)

Tolerance $=10.0 \mathrm{PPM} / \mathrm{DBE}: \min =-1.5, \max =50.0$

Selected filters: None

Monoisotopic Mass, Odd and Even Electron lons

9 formula(e) evaluated with 1 results within limits (up to 50 closest results for each mass)

Elements Used:

$\begin{array}{llll}C: 0-200 & H: 0-400 & 0: 4-6 & F: 3-3\end{array} \quad$ S: $1-1$

ZZCCC4

10-Jul-202009:35:03

352.0597
100

Autospec
Voltage El+
3

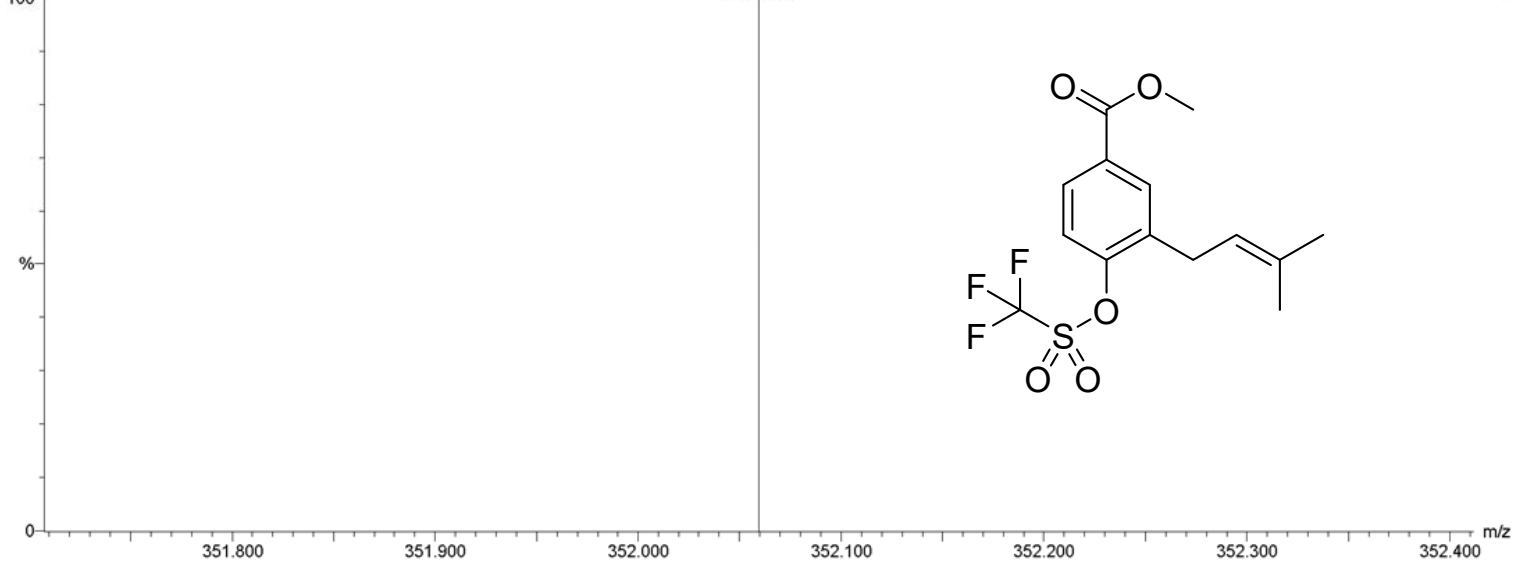

$\begin{array}{lll}\text { Minimum: } & 500.0 \quad 10.0 & -1.5 \\ \text { Maximum: } & 50.0\end{array}$

Mass Calc. Mass mDa PPM DBE i-FIT Formula

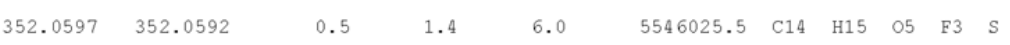


IR spectrum of compound $\mathbf{5}$.

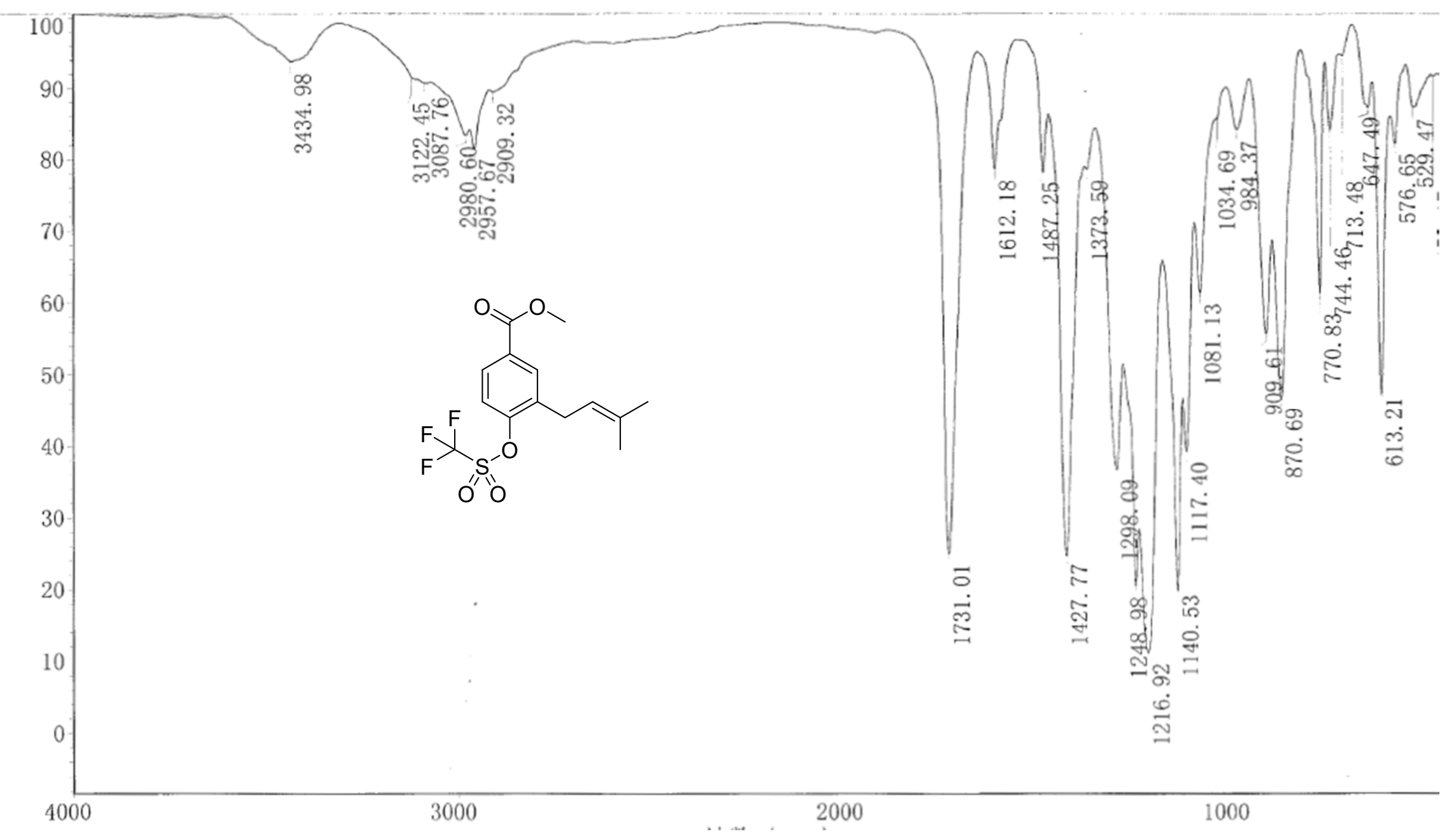


${ }^{1} \mathrm{H}$ NMR spectrum (400 MHz, $\mathrm{CDCl}_{3}$ ) of compound 6 .

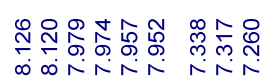
更证
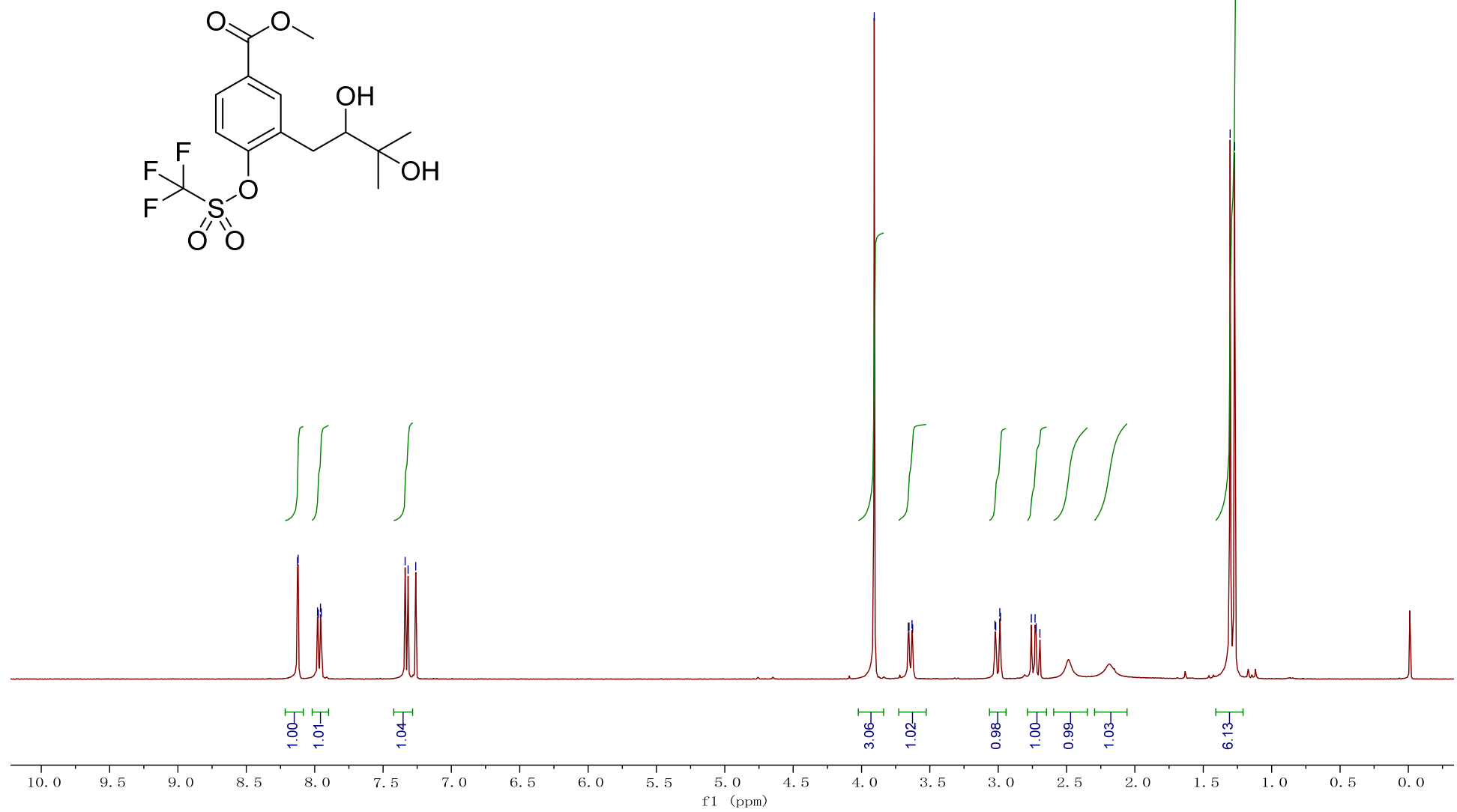
${ }^{13} \mathrm{C}$ and DEPT NMR spectra $\left(100 \mathrm{MHz}, \mathrm{CDCl}_{3}\right)$ of compound 6.
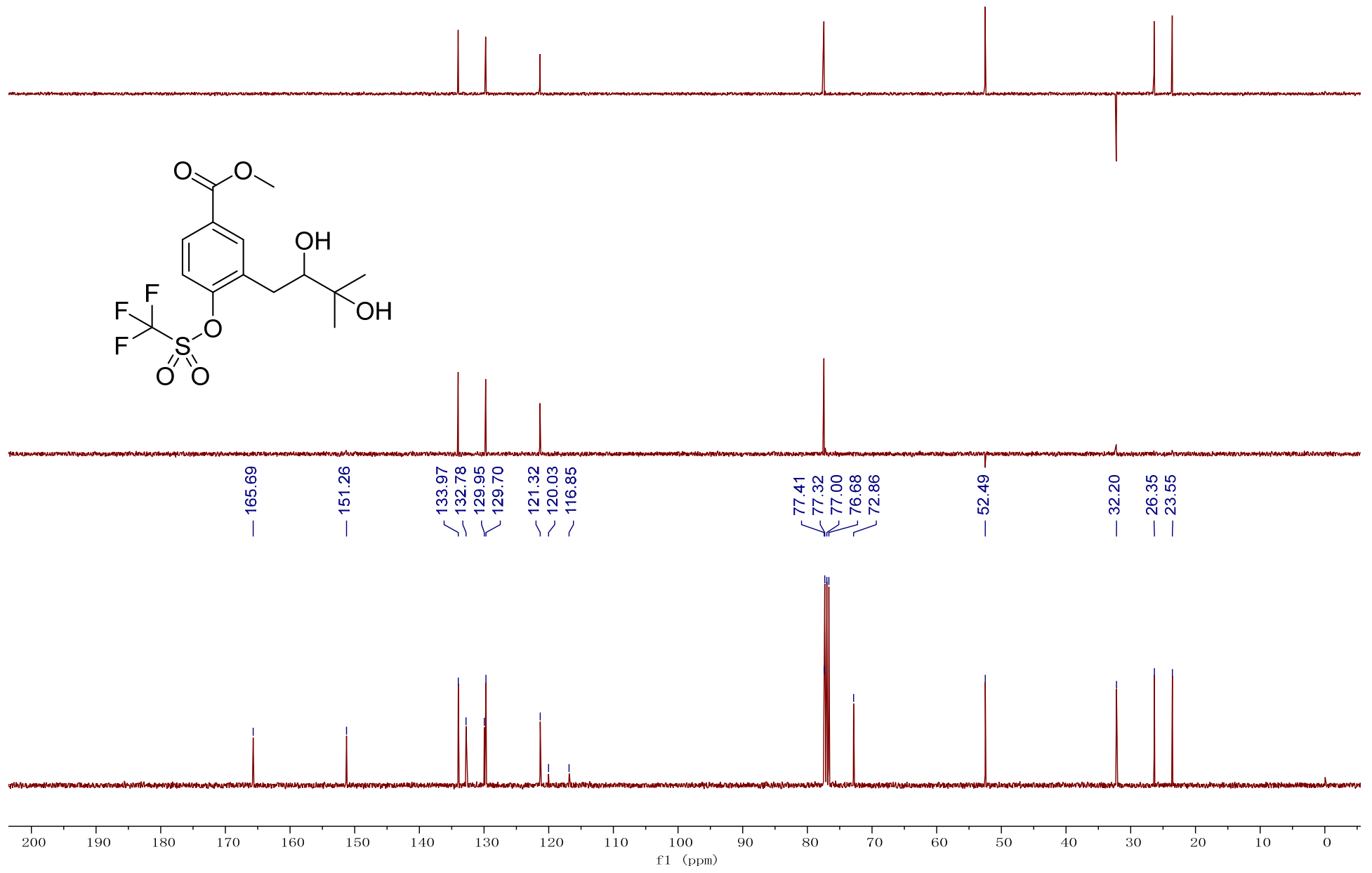

S29 
HRESIMS spectrum of compound 6 .

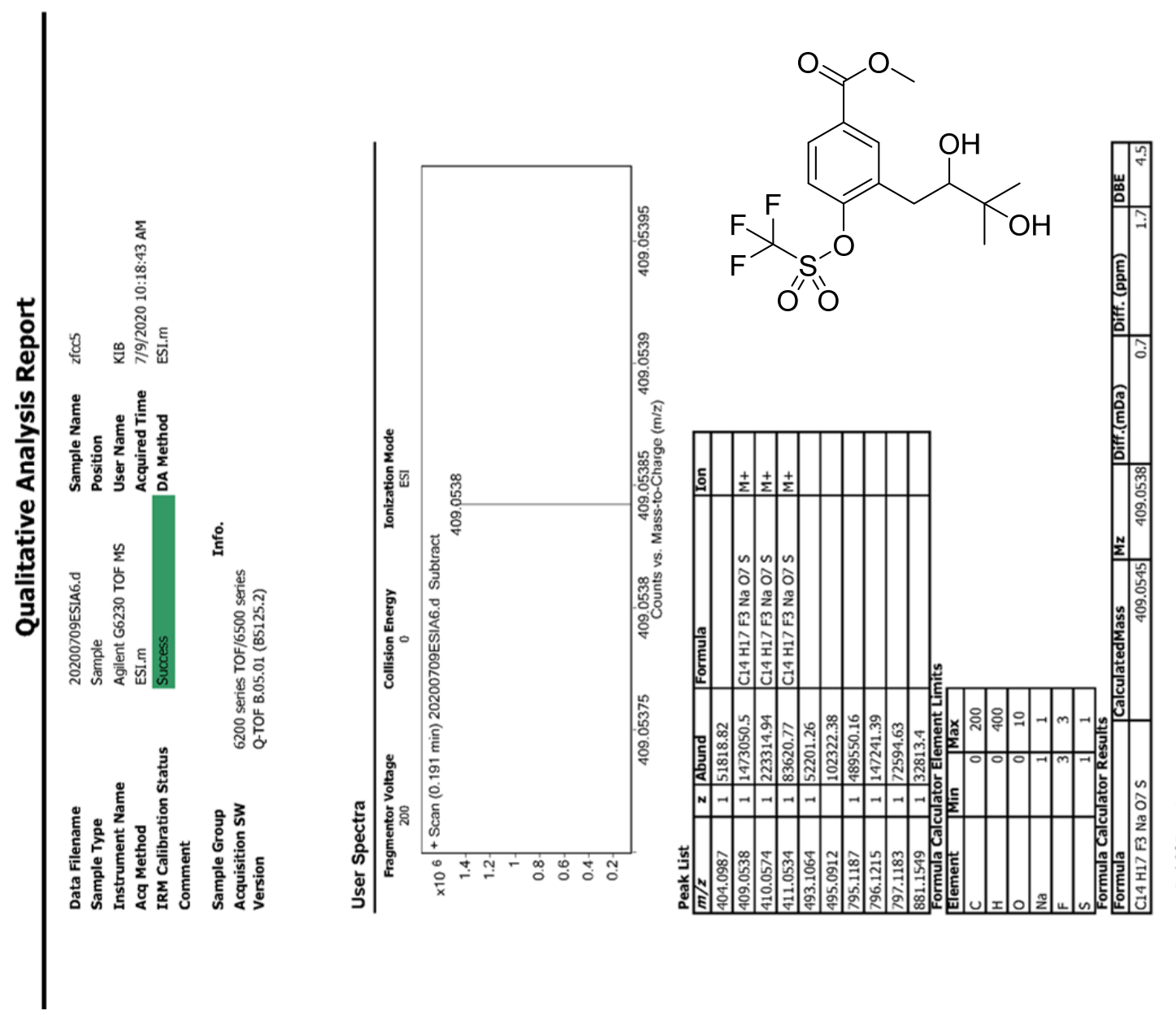


IR spectrum of compound $\mathbf{6}$.

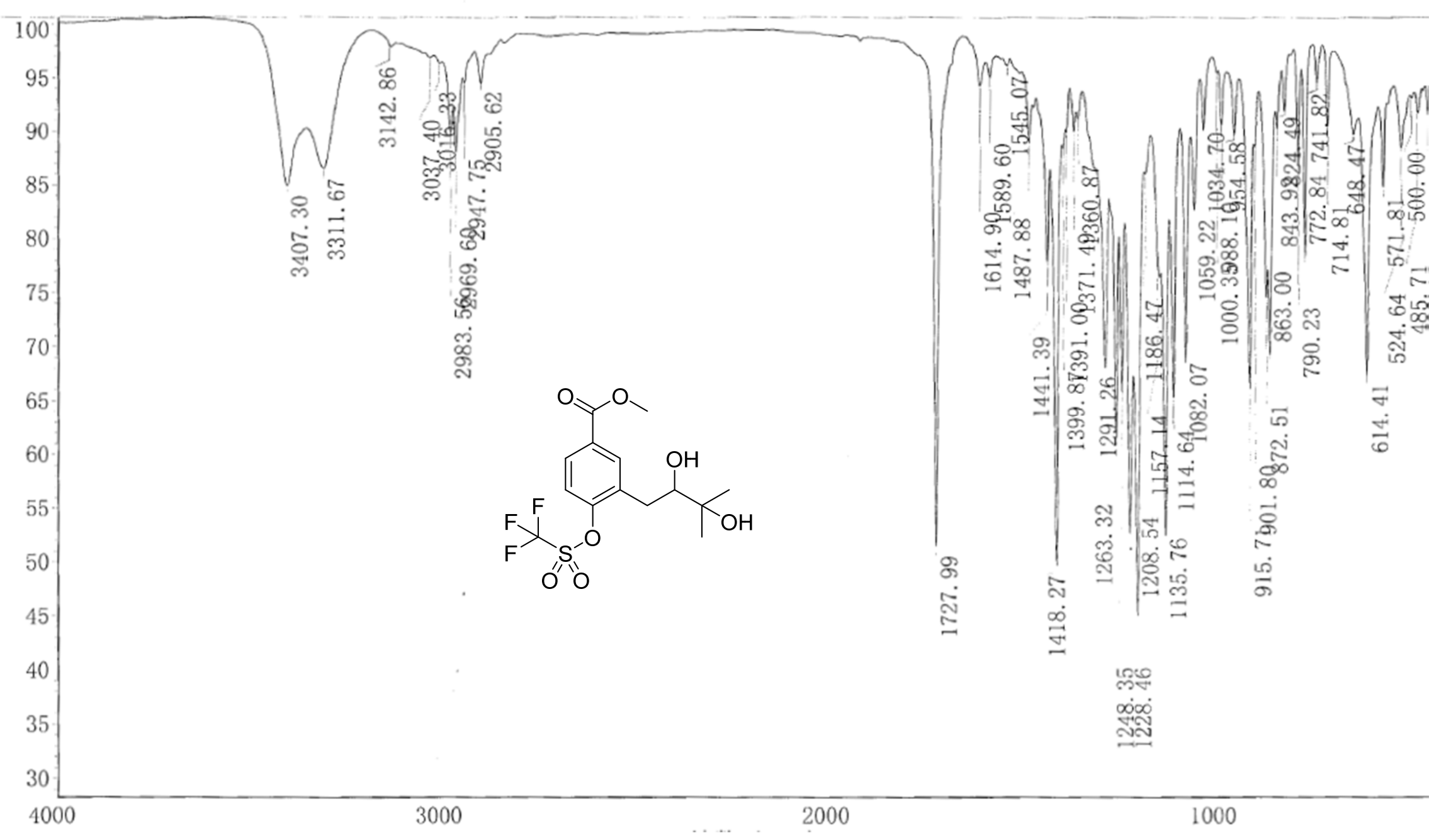


${ }^{1} \mathrm{H}$ NMR spectrum $\left(400 \mathrm{MHz}, \mathrm{CDCl}_{3}\right)$ of compound 7.

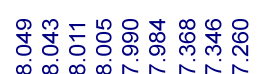
$\underbrace{\infty} \underbrace{\infty} \infty \pi N$

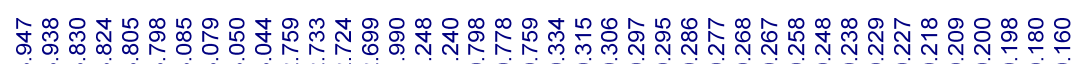

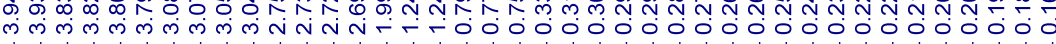
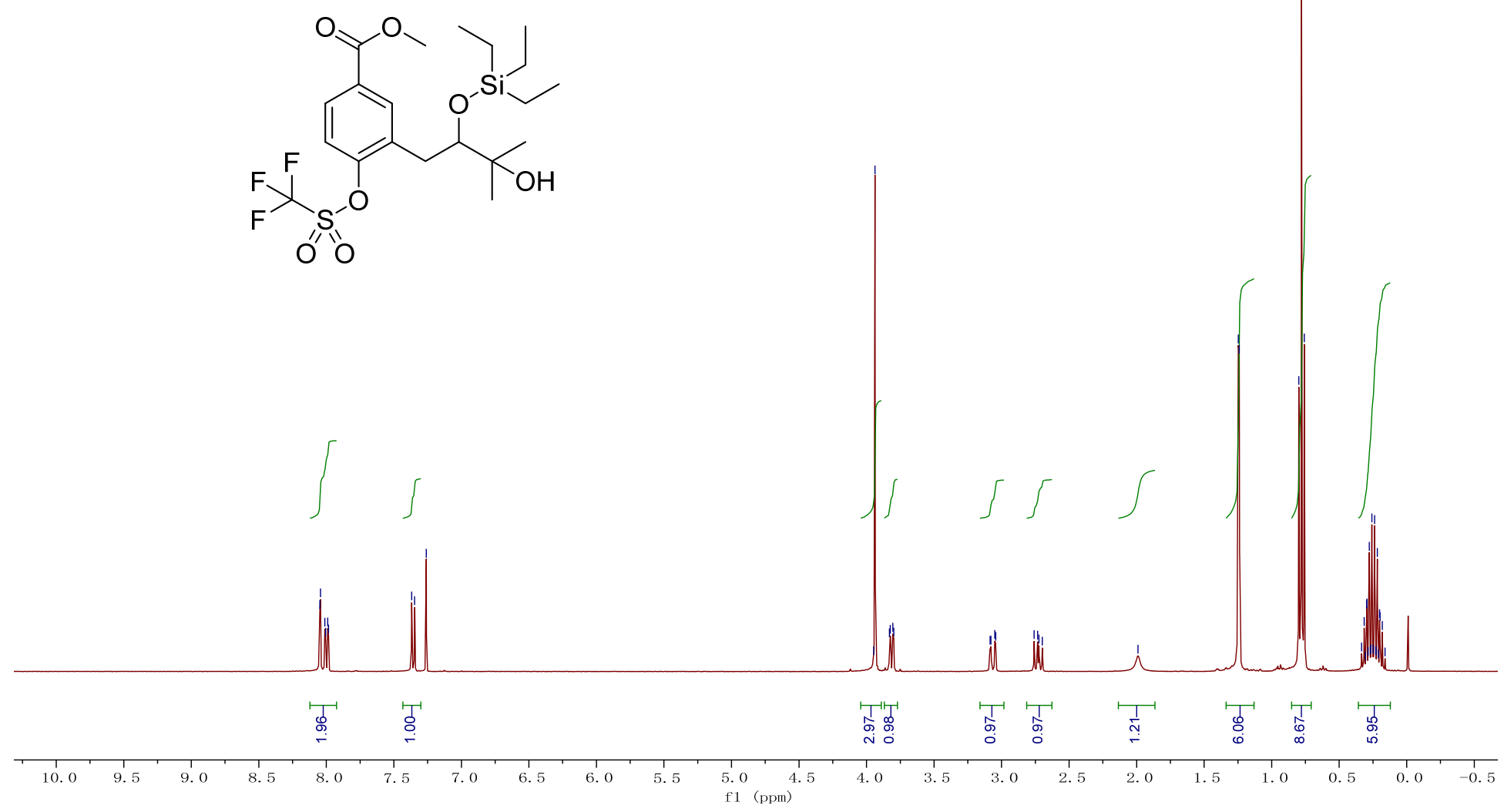
${ }^{13} \mathrm{C}$ and DEPT NMR spectra (100 MHz, $\left.\mathrm{CDCl}_{3}\right)$ of compound 7.

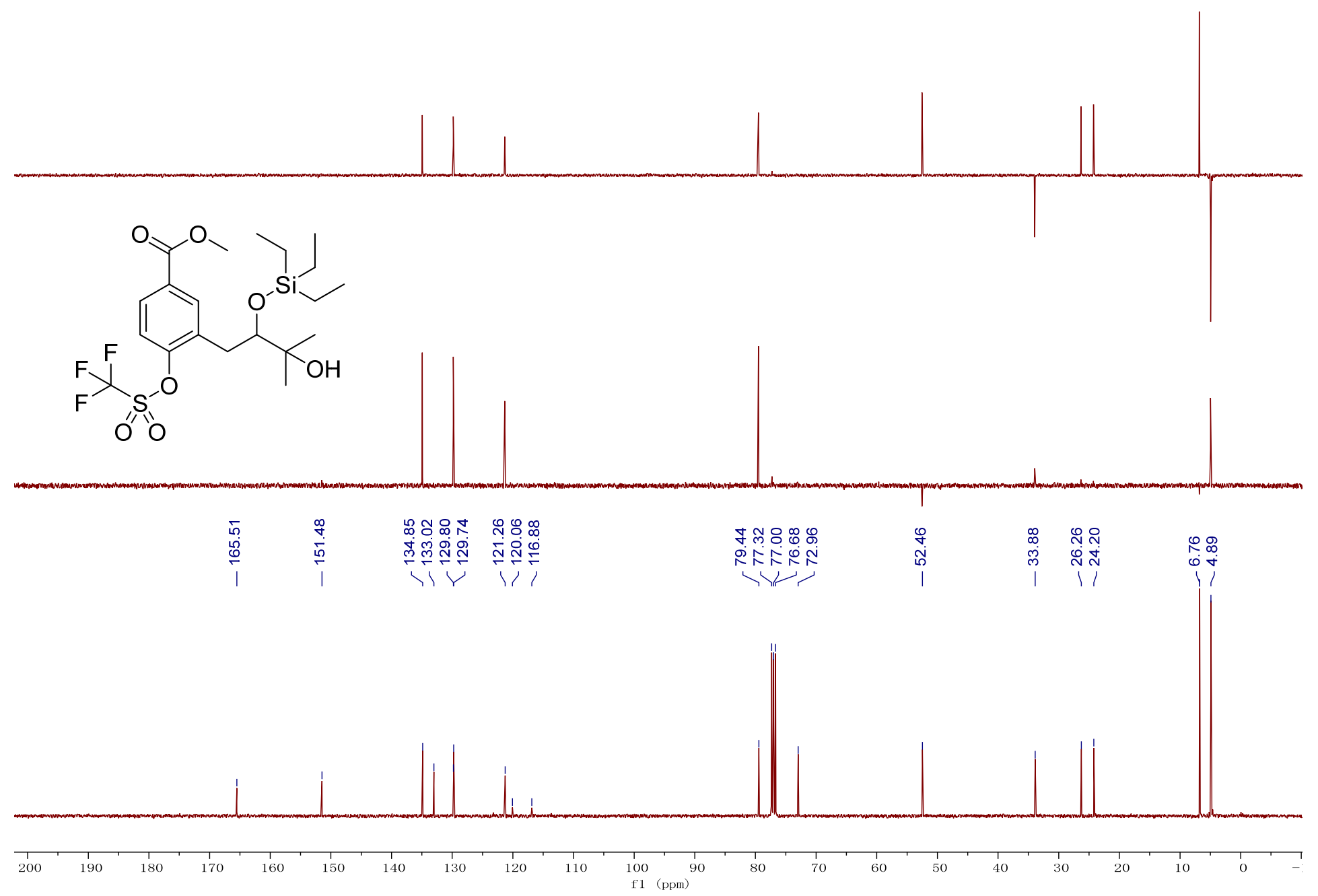


HREIMS spectrum of compound 7.

\section{Elemental Composition Report}

Page 1

Single Mass Analysis (displaying only valid results)

Tolerance $=10.0$ PPM / DBE: $\min =-1.5, \max =50.0$

Selected filters: None

Monoisotopic Mass, Odd and Even Electron lons

13 formula (e) evaluated with 1 results within limits (up to 50 closest results for each mass)

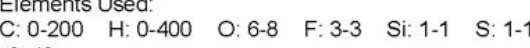

Zicc10 10 10-Jul-202009:22:40

500.1503

Autospec
Voltage $E_{1+1}$
2.11

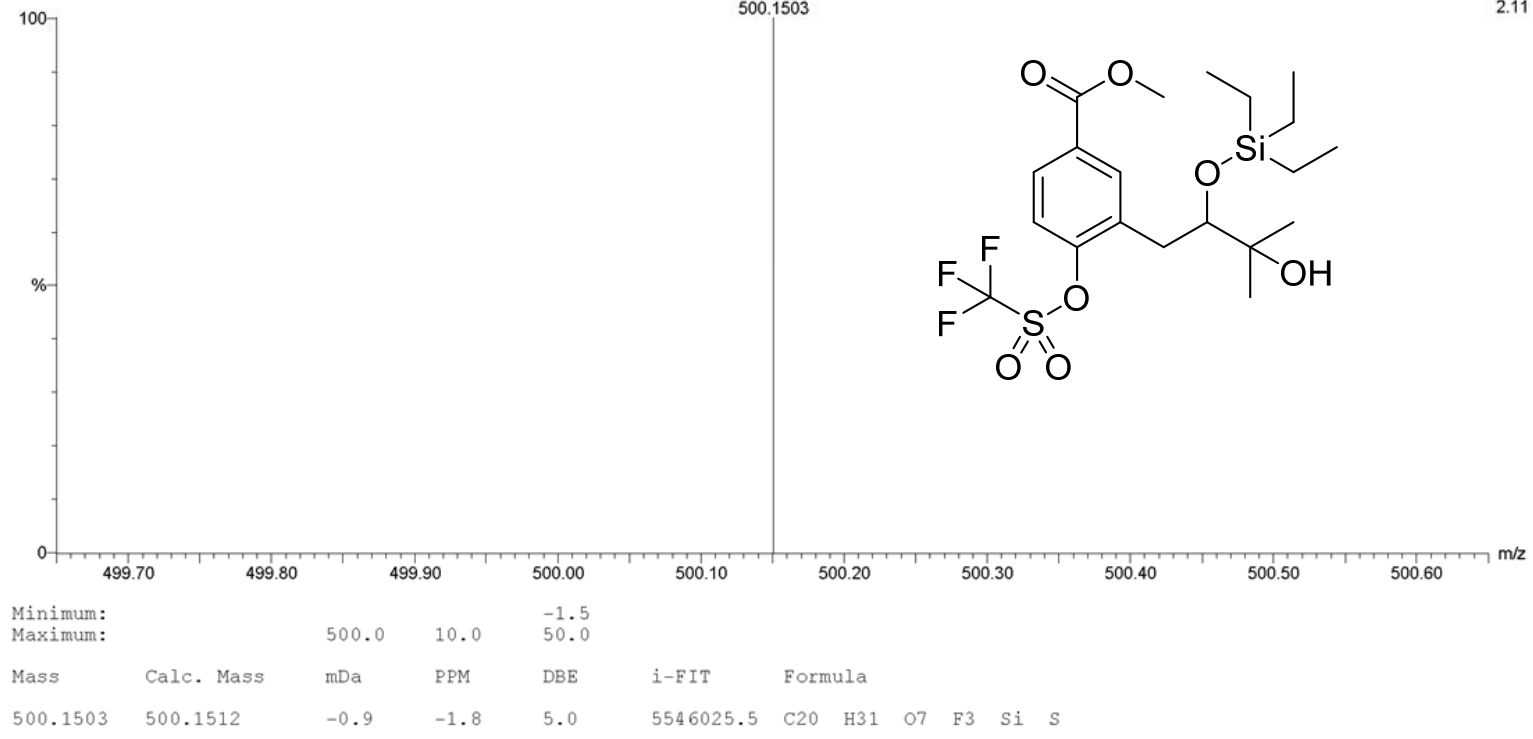


IR spectrum of compound 7.

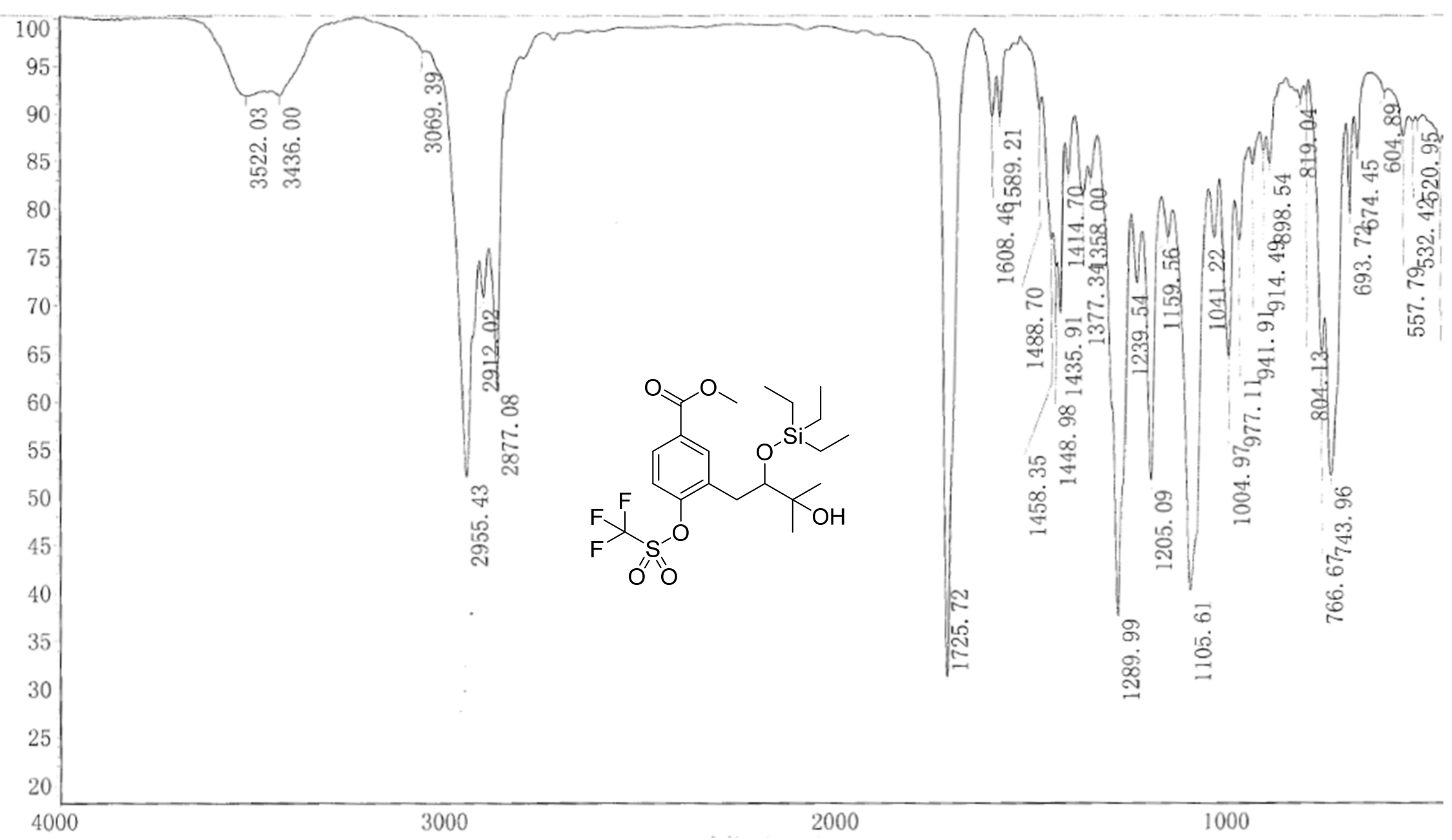


${ }^{1} \mathrm{H}$ NMR spectrum $\left(400 \mathrm{MHz}, \mathrm{CDCl}_{3}\right)$ of compound 8 .

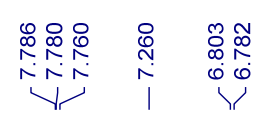
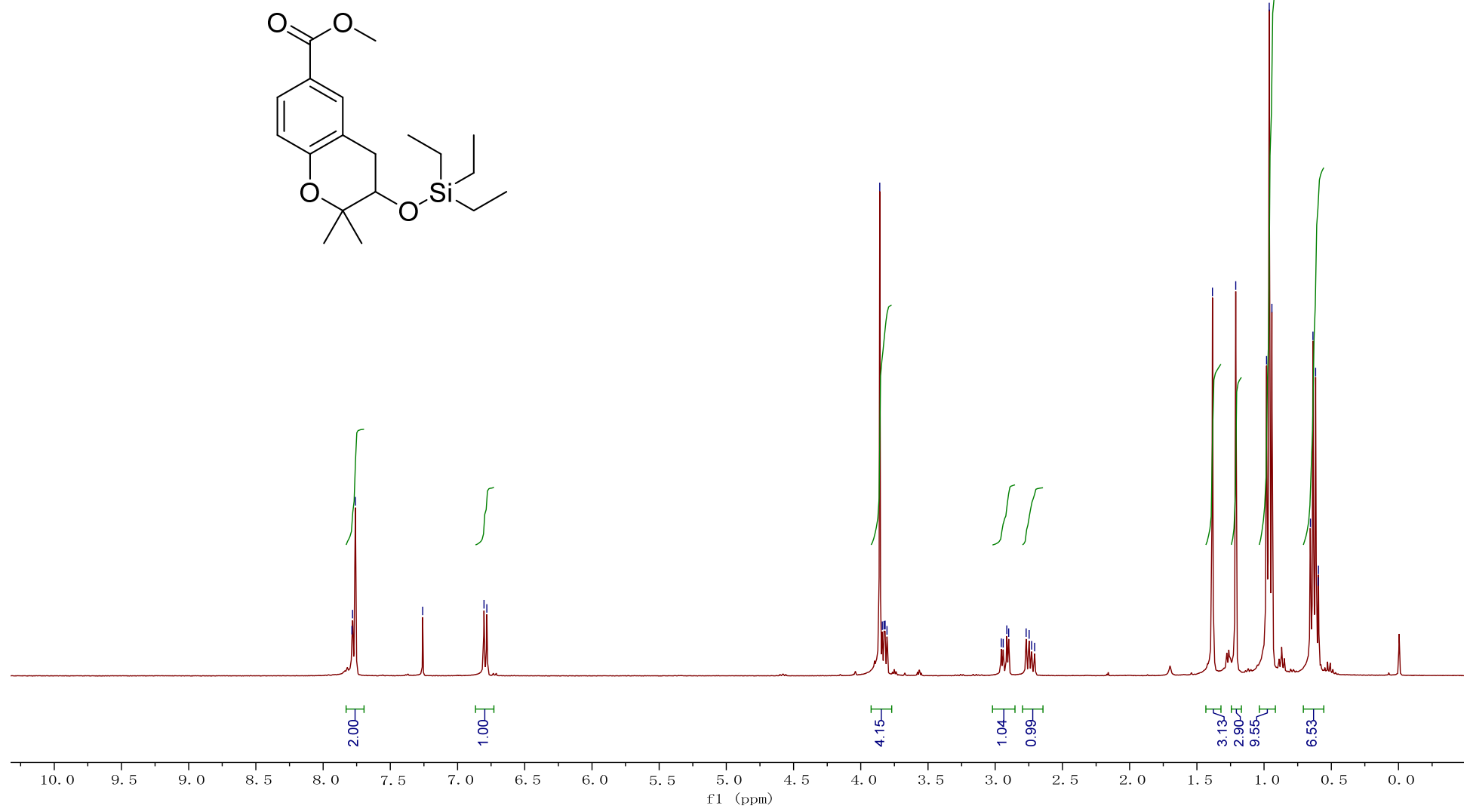
${ }^{13} \mathrm{C}$ and DEPT NMR spectra (100 MHz, $\left.\mathrm{CDCl}_{3}\right)$ of compound 8 .
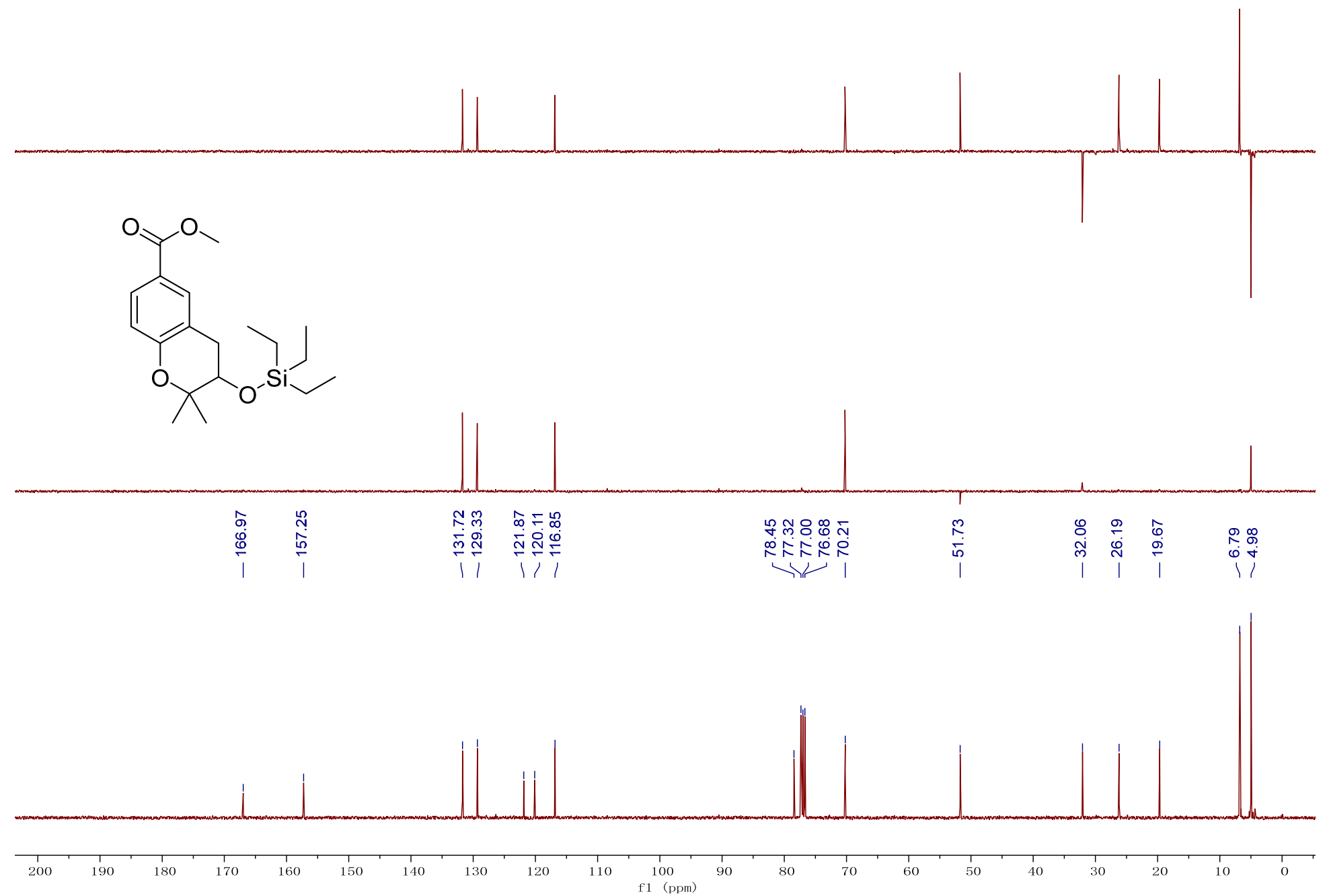
HRESIMS spectrum of compound $\mathbf{8}$.

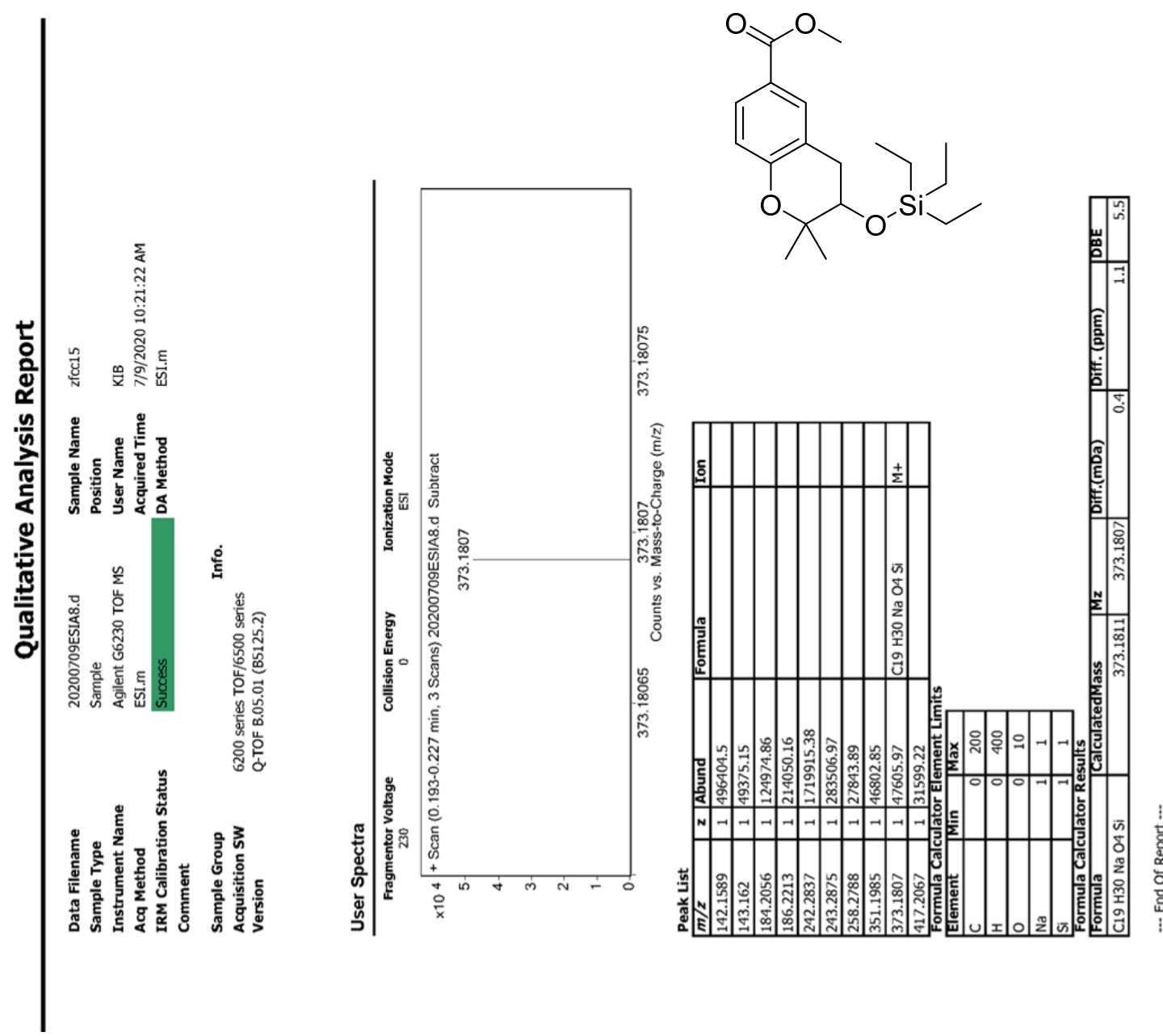


HRESIMS spectrum of compound $\mathbf{8}$.

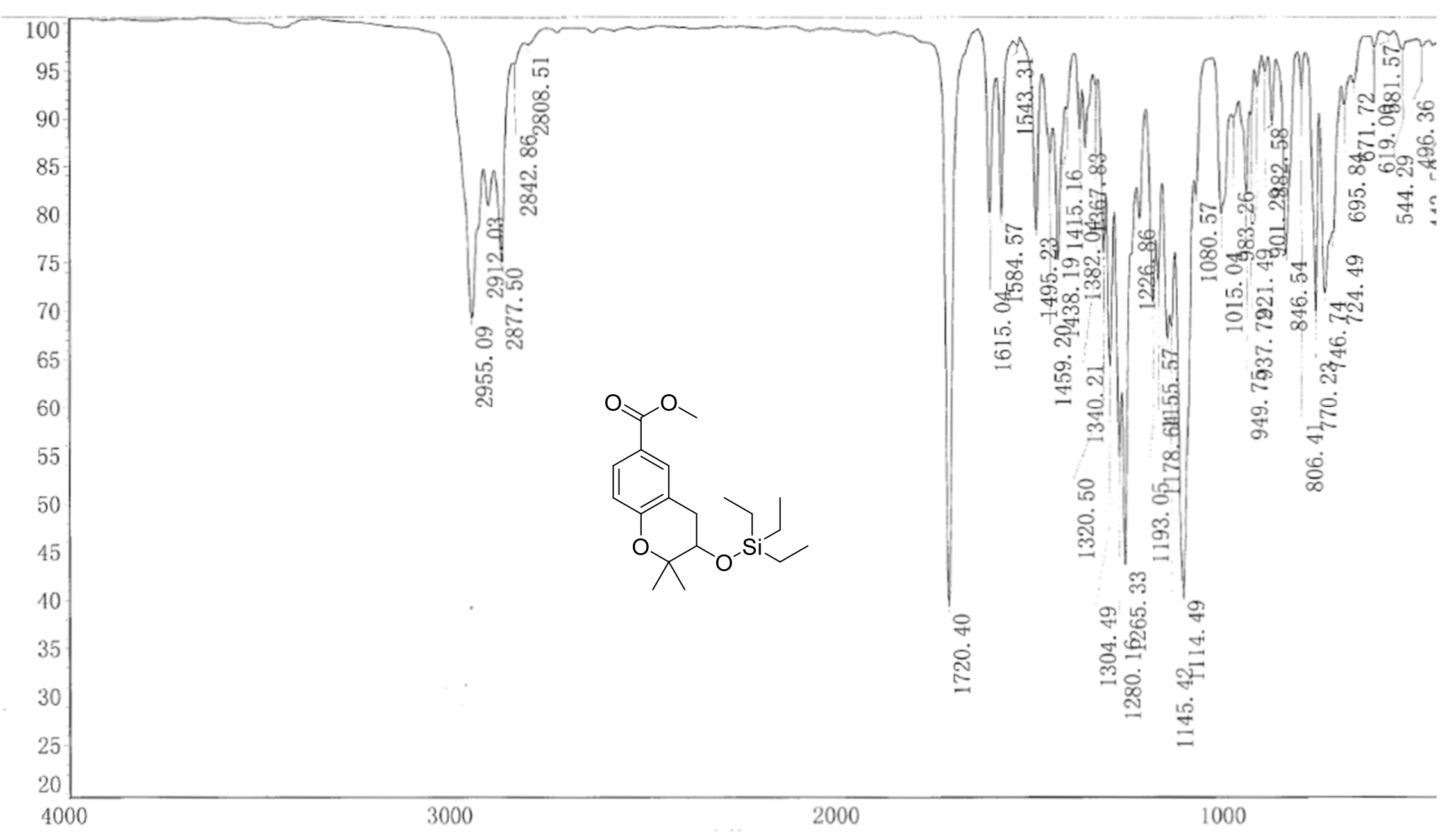


${ }^{1} \mathrm{H}$ NMR spectrum (400 MHz, $\mathrm{CDCl}_{3}$ ) of compound 9.

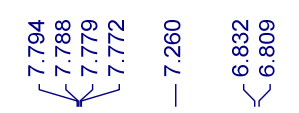

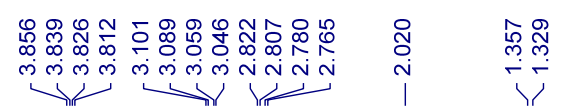
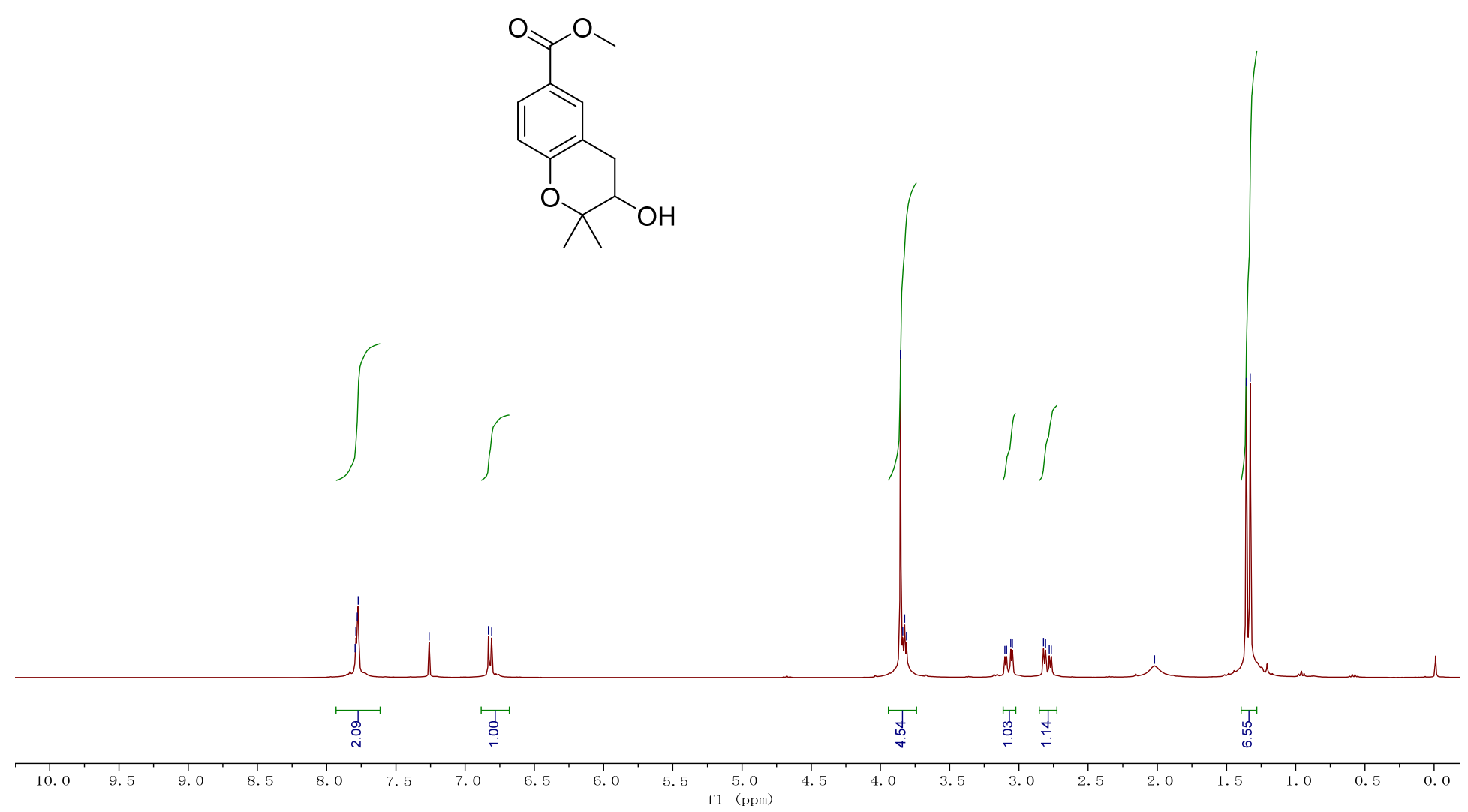
${ }^{13} \mathrm{C}$ and DEPT NMR spectra $\left(100 \mathrm{MHz}, \mathrm{CDCl}_{3}\right)$ of compound 9.

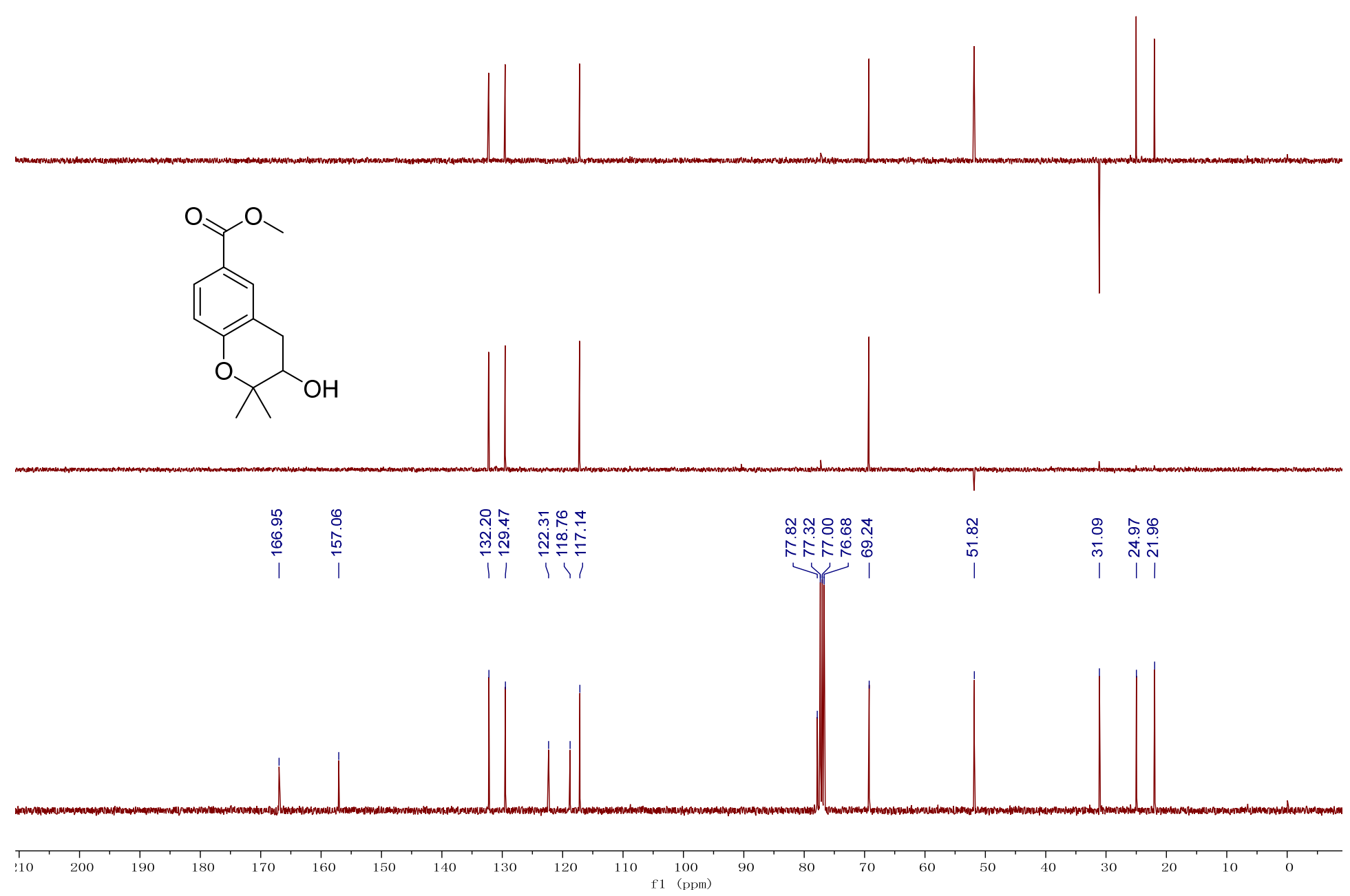


${ }^{1} \mathrm{H}$ NMR spectrum $\left(400 \mathrm{MHz}, \mathrm{CDCl}_{3}\right)$ of compound $\mathbf{1 0}$.
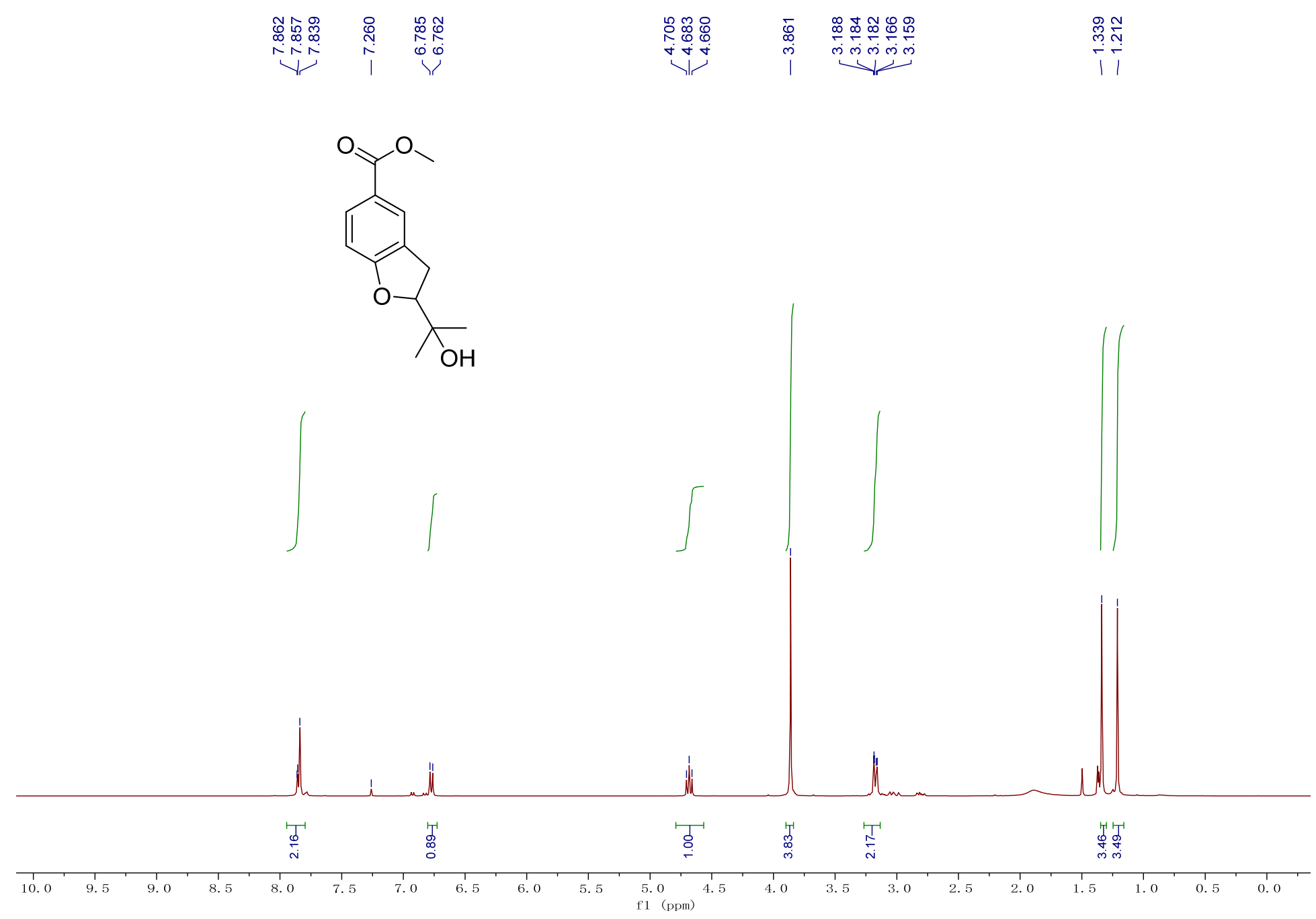
${ }^{13} \mathrm{C}$ and DEPT NMR spectra $\left(100 \mathrm{MHz}, \mathrm{CDCl}_{3}\right)$ of compound $\mathbf{1 0}$.

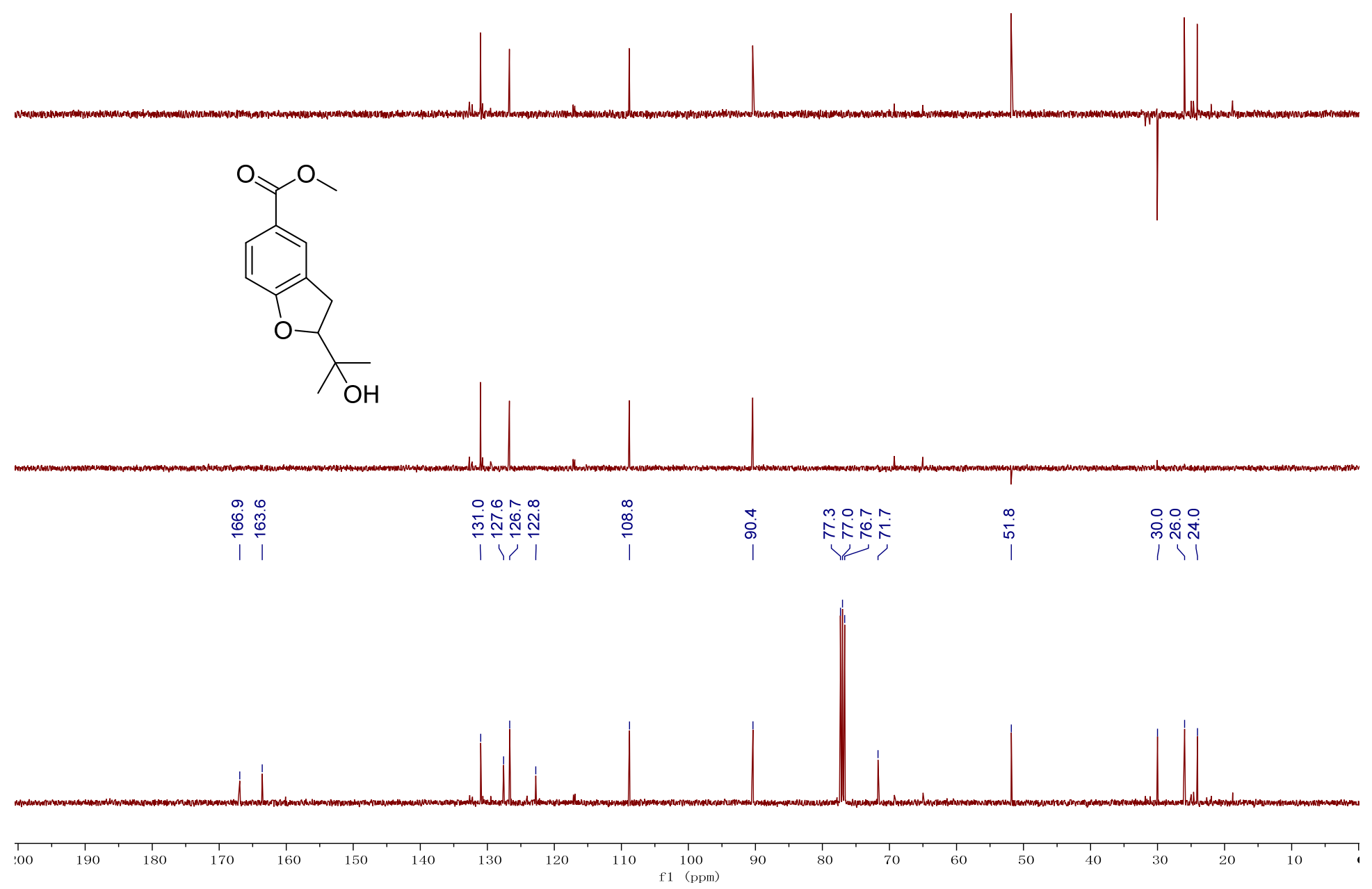




\section{References}

(1) Passreiter, C. M.; Suckow-Schnitker, A. K.; Kulawik, A.; Addae-Kyereme, J.; Wright, C. W.; Waetjen, W., Prenylated flavanone derivatives isolated from Erythrina addisoniae are potent inducers of apoptotic cell death. Phytochemistry 2015, 117, $237-244$.

(2) Cui, L.; Thuong, P. T.; Lee, H. S.; Ndinteh, D. T.; Mbafor, J. T.; Fomum, Z. T.; Oh, W. K., Flavanones from the stem bark of Erythrina abyssinica. Bioorg. Med. Chem. 2008, 16, 10356-10362.

(3) Syah, Y. M.; Ghisalberti, E. L., Flavanone derivatives from Macaranga tanarius. Biochem. Syst. Ecol. 2015, 62, $151-154$.

(4) Tseng, M. H.; Chou, C. H.; Chen, Y. M.; Kuo, Y. H., Allelopathic prenylflavanones from the fallen leaves of Macaranga tanarius. J. Nat. Prod. 2001, 64, 827-828.

(5) Yao C. S.; Zhang X. H.; Zhang W.; Shen Y. H.; Xu Y. L., New flavonol glycosides from Epimedium sagittatum. Nat. Prod. Res. Dev. 2004, $16,101-103$.

(6) Zhang, Y. L.; Luo, J. G.; Wan, C. X.; Zhou, Z. B.; Kong, L. Y., Four new flavonoids with $\alpha$-glucosidase inhibitory activities from Morus alba var. tatarica. Chem. Biodivers. 2015, 12, 1768-1776.

(7) Zheng, Z. F.; Zhang, Q. J.; Chen, R. Y.; Yu, D. Q., Four new flavonoids from Morus australis. J. Asian Nat. Prod. Res. 2012, 14, $263-269$.

(8) Tian, X. M.; Chen, S. Z.; Tang, L.; Tu, P. F., Three new isoflavonoids from the aerial parts of Ammopiptanthus mongolicus. Helv. Chim. Acta 2008, 91, 1015-1022.

(9) Daus, M.; Chaithada, P.; Phongpaichit, S.; Watanapokasin, R.; Carroll, A. R.; Mahabusarakam, W., New prenylated dihydrochalcones from the leaves of Artocarpus elasticus. Phytochem. Lett. 2017, 19, 226-230. 
(10) Baek, Y. S.; Ryu, Y. B.; Curtis-Long, M. J.; Ha, T. J.; Rengasamy, R.; Yang, M. S.; Park, K. H., Tyrosinase inhibitory effects of 1,3-diphenylpropanes from Broussonetia kazinoki. Bioorg. Med. Chem. 2009, 17, 35-41.

(11) Gibbons, S.; Moser, E.; Hausmann, S.; Stavri, M.; Smith, E.; Clennett, C., An anti-staphylococcal acylphloroglucinol from Hypericum foliosum. Phytochemistry 2005, 66, 1472-1475.

(12) Fuller, R. W.; Westergaard, C. K.; Collins, J. W.; Cardellina, J. H.; Boyd, M. R., Vismiaphenones D-G, new prenylated benzophenones from Vismia cayennensis. J. Nat. Prod. 1999, 62, 67-69.

(13) Hauschild, W.; Mutiso, P. B. C.; Passreiter, C. M., Prenylated pterocarpanes from Erythrina melanacantha. Nat. Prod. Commun. 2010, 5, $721-724$.

(14) Tanjung, M.; Hakim, E. H.; Syah, Y. M., Prenylated dihydrostilbenes from Macaranga rubiginosa. Chem. Nat. Compd. 2017, 53, 215-218.

(15) Jeong, S. H.; Ryu, Y. B.; Curtis-Long, M. J.; Ryu, H. W.; Baek, Y. S.; Kang, J. E.; Lee, W. S.; Park, K. H., Tyrosinase inhibitory polyphenols from roots of Morus Ihou. J. Agric. Food Chem. 2009, 57, 1195-1203.

(16) Wang, Y. N.; Liu, M. F.; Hou, W. Z.; Xu, R. M.; Gao, J.; Lu, A. Q.; Xie, M. P.; Li, L.; Zhang, J. J.; Peng, Y.; Ma, L. L.; Wang, X. L.; Shi, J. G.; Wang, S. J., Bioactive benzofuran derivatives from Cortex mori radicis, and their neuroprotective and analgesic activities mediated by mGluR $_{1}$. Molecules 2017, 22, 236.

(17) Asakawa, Y.; Kondo, K.; Tori, M.; Hashimoto, T.; Ogawa, S., Prenyl bibenzyls from the liverwort Radula kojana. Phytochemistry 1991, 30, 219-234.

(18) Toyota, M.; Shimamura, T.; Ishii, H.; Renner, M.; Braggins, J.; Asakawa, Y., New bibenzyl cannabinoid from the New Zealand liverwort 
Radula marginata. Chem. Pharm. Bull. 2002, 50, 1390-1392.

(19) Zeng, Q.; Zhong, W. M.; Chen, Y. C.; Xiang, Y.; Chen, X. Y.; Tian, X. P.; Zhang, W. M.; Zhang, S.; Wang, F. Z., A new butenolide derivative from the deep-sea fungus Aspergillus terreus SCSIO FZQ028. Nat. Prod. Res. 2019, 1-8.

(20) Zhang, Y. L.; Luo, J. G.; Wan, C. X.; Zhou, Z. B.; Kong, L. Y., Geranylated 2-arylbenzofurans from Morus alba var. tatarica and their $\alpha$-glucosidase and protein tyrosine phosphatase 1B inhibitory activities. Fitoterapia 2014, 92, 116-126.

(21) Radwan, M. M.; Ross, S. A.; Slade, D.; Ahmed, S. A.; Zulfiqar, F.; ElSohly, M. A., Isolation and characterization of new cannabis constituents form high potency variety. Planta Med. 2008, 74, 267-272.

(22) Cheng, Y. B.; Fazary, A. E.; Lin, Y. C.; Lo, I. W.; Ong, S. C.; Chen, S. Y.; Chien, C. T.; Lin, Y. J.; Lin, W. W.; Shen, Y. C., Hyperinakin, a new anti-inflammatory phloroglucinol derivative from Hypericum nakamurai. Nat. Prod. Res. 2013, 27, 727-734.

(23) Innok, P.; Rukachaisirikul, T.; Phongpaichit, S.; Suksamrarn, A., Fuscacarpans A-C, new pterocarpans from the stems of Erythrina fusca. Fitoterapia 2010, 81, 518-523.

(24) Tanaka, H.; Tanaka, T.; Etoh, H., Three pterocarpans from Erythrina crista-galli. Phytochemistry 1997, 45, 835-838.

(25) Magadula, J. J.; Kapingu, M. C.; Mbwambo, Z. H.; Mulholland, D. A., Secondary metabolites from Teclea amanuensis (Rutaceae) from Tanzania. Nat. Prod. Commun. 2008, 3, 1683-1686.

(26) Kiplimo, J. J.; Koorbanally, N. A., Antibacterial activity of an epoxidised prenylated cinnamaldehdye derivative from Vepris glomerata. Phytochem. Lett. 2012, 5, 438-442.

(27) Ishii, H.; Ishikawa, T.; Chen, I. S.; Lu, S. T., New phenyl propanoids having weak piscicidal action and the related compound: wutaiensol, 
wutaiensal, wutaialdehyde, and methyl demethoxywutaiensate. Tetrahedron Lett. 1982, 23, 4345-4348.

(28) Piacente, S.; Aquino, R.; Detommasi, N.; Deugaz, O. L.; Orellana, H. C., p-hydroxyacetophenone derivatives from Werneria ciliolata. Phytochemistry 1992, 31, 2182-2184.

(29) Paredes, A.; Palacios, J.; Quispe, C.; Nwokocha, C. R.; Morales, G.; Kuzmicic, J.; Cifuentes, F., Hydroalcoholic extract and pure compounds from Senecio nutans Sch. Bip (Compositae) induce vasodilation in rat aorta through endothelium-dependent and independent mechanisms. J. Ethnopharmacol. 2016, 192, 99-107.

(30) Zhao, M. B.; Zhou, S. X.; Zhang, Q. Y.; Wei, W. F.; Li, M. H.; Xing, J. Y.; Jiang, Y.; Tu, P. F., Prenylated benzoic acid derivatives from the stem of Euodia lepta. Nat. Prod. Res. 2017, 31, 1589-1593.

(31) Lago, J. H. G.; Ramos, C. S.; Casanova, D. C. C.; Morandim, A. D.; Bergamo, D. C. B.; Cavalheiro, A. J.; Bolzani, V. D.; Furlan, M.; Guimaraes, E. F.; Young, M. C. M.; Kato, M. J., Benzoic acid derivatives from Piper species and their fungitoxic activity against Cladosporium cladosporioides and C. sphaerospermum. J. Nat. Prod. 2004, 67, 1783-1788.

(32) Arriaga-Giner, F. J.; Wollenweber, E.; Schober, I.; Yatskievych, G., Three new benzoic acid derivatives from the glandular excretion of Eriodictyon sessilifolium (Hydrophyllaceae). Z. Naturforsch., C: Biosci. 1988, 43, 337-340.

(33) Kapche, G. D. W. F.; Fozing, C. D.; Donfack, J. H.; Fotso, G. W.; Amadou, D.; Tchana, A. N.; Bezabih, M.; Moundipa, P. F.; Ngadjui, B. T.; Abegaz, B. M., Prenylated arylbenzofuran derivatives from Morus mesozygia with antioxidant activity. Phytochemistry 2009, 70, $216-221$.

(34) Kaur, N.; Xia, Y.; Jin, Y.; Dat, N. T.; Gajulapati, K.; Choi, Y.; Hong, Y.-S.; Lee, J. J.; Lee, K., The first total synthesis of moracin O and moracin P, and establishment of the absolute configuration of moracin O. Chem. Commun. 2009, 1879-1881. 
(35) Pailee, P.; Sangpetsiripan, S.; Mahidol, C.; Ruchirawat, S.; Prachyawarakorn, V., Cytotoxic and cancer chemopreventive properties of prenylated stilbenoids from Macaranga siamensis. Tetrahedron 2015, 71, 5562-5571.

(36) Shaffer, C. V.; Cai, S.; Peng, J.; Robles, A. J.; Hartley, R. M.; Powell, D. R.; Du, L.; Cichewicz, R. H.; Mooberry, S. L., Texas native plants yield compounds with cytotoxic activities against prostate cancer cells. J. Nat. Prod. 2016, 79, 531-540.

(37) Subrahmanyam, K.; Rao, J. M.; Vemuri, V. S. S.; Babu, S. S.; Roy, C. P.; Rao, K. V. J.; Merlini, L., New chalcones from leaves of Flemingia stricta Roxb. (Leguminosae). Indian J. Chem., Sect. B 1982, 21, 895-897.

(38) Jung, D. J.; Porzel, A.; Huneck, S., Gigasol and other coumarins from Angelica gigas. Phytochemistry 1991, 30, 710-712.

(39) Blunt, S. B.; Chen, T. B.; Wiemer, D. F., Prenylated benzoic acids from Rapanea myricoides. J. Nat. Prod. 1998, 61, 1400-1403.

(40) Hirota, M.; Miyazaki, S.; Minakuchi, T.; Takagi, T.; Shibata, H., Myrsinoic acids B, C and F, anti-inflammatory compounds from Myrsine seguinii. Biosci., Biotechnol., Biochem. 2002, 66, 655-659.

(41) Urones, J. G.; Teresa, J. D.; Marcos, I. S.; Moro, R. F.; Barcala, P. B.; Cuadrado, M. J. S., Acetophenones and terpenoids from Senecio Gallicus. Phytochemistry 1987, 26, 1113-1115.

(42) Lai, P. X.; Ma, Q. L.; Row, K. H., A new acetophenone derivative and other constituents from Senecio vulgaris. J. Chem. Res. 2010, 34, $514-516$.

(43) Liu, G. Y.; Shuang, C.; Zhang, Y. M.; Xu, J. M.; Lin, R. C., Study on chemical constituents in seeds of Helicia nilagirica (II). Chin. J. Chin. Mater. Med. 2005, 30, 830-832.

(44) Quader, M. A.; Elturbi, J. A.; Armstrong, J. A.; Gray, A. I.; Waterman, P. G., Coumarins and their taxonomic value in the genus Phebalium. 
Phytochemistry 1992, 31, 3083-3089.

(45) Deachathai, S.; Phongpaichit, S.; Mahabusarakam, W., Phenolic compounds from the seeds of Garcinia dulcis. Nat. Prod. Res. 2008, 22, $1327-1332$.

(46) Nilar; Harrison, L. J., Xanthones from the heartwood of Garcinia mangostana. Phytochemistry 2002, 60, 541-548.

(47) Cui, L.; Lee, H. S.; Ndinteh, D. T.; Mbafor, J. T.; Kim, Y. H.; Le, T. V. T.; Nguyen, P. H.; Oh, W. K., New prenylated flavanones from Erythrina abyssinica with protein tyrosine phosphatase 1B (PTP1B) inhibitory activity. Planta Med. 2010, 76, 713-718.

(48) Nuclear, P.; Sommit, D.; Boonyuen, N.; Pudhom, K., Butenolide and furandione from an endophytic Aspergillus terreus. Chem. Pharm. Bull. 2010, 58, 1221-1223.

(49) Rao, K. V.; Sadhukhan, A. K.; Veerender, M.; Ravikumar, V.; Mohan, E. V. S.; Dhanvantri, S. D.; Sitaramkumar, M.; Babu, J. M.; Vyas, K.; Reddy, G. O., Butyrolactones from Aspergillus terreus. Chem. Pharm. Bull. 2000, 48, 559-562.

(50) Wang, H.; Leach, D. N.; Forster, P. I.; Thomas, M. C.; Blanksby, S. J.; Waterman, P. G., Prenylated bisresorcinols from Grevillea floribunda. Phytochem. Lett. 2009, 2, 41-45.

(51) Xia, Y.; Jin, Y.; Kaur, N.; Choi, Y.; Lee, K., Synthesis and biological evaluation of novel moracin O and P analogues. Eur. J. Med. Chem. 2011, 46, 2386-2396.

(52) Tan, Y.-X.; Yang, Y.; Zhang, T.; Chen, R.-Y.; Yu, D.-Q., Bioactive 2-arylbenzofuran derivatives from Morus wittiorum. Fitoterapia 2010, 81, $742-746$.

(53) Hu, X.; Wang, M.; Yan, G. R.; Yu, M. H.; Wang, H. Y.; Hou, A. J., 2-Arylbenzofuran and tyrosinase inhibitory constituents of Morus 
notabilis. J. Asian Nat. Prod. Res. 2012, 14, 1103-1108.

(54) Wang, L.; Yang, Y.; Liu, C.; Chen, R.-Y., Three new compounds from Morus nigra L. J. Asian Nat. Prod. Res. $2010,12,431-437$.

(55) Takasugi, M.; Nagao, S.; Munoz, L.; Ishikawa, S.; Masamune, T.; Shirata, A.; Takahashi, K. The structure of phytoalexins produced in diseased mulberry. Koen Yoshishu-Tennen Yuki Kagobutsu Toronkai 1979, 22, 275-282.

(56) Wen, R.; Lv, H. N.; Jiang, Y.; Tu, P. F., Anti-inflammatory flavone and chalcone derivatives from the roots of Pongamia pinnata (L.) Pierre. Phytochemistry 2018, 149, 56-63.

(57) Moriyasu, M.; Ichimaru, M.; Nishiyama, Y.; Kato, A.; Mathenge, S. G.; Juma, F. D.; Nganga, J. N., Minor flavanones from Erythrina abyssinica. J. Nat. Prod. 1998, 61, 185-188.

(58) Peng, W. W.; Yan, H.; Tan, N. H., One new chroman glycoside derivative from unmatured fruits of Citrus aurantium. Chin. J. Chin. Mater. Med. 2013, 38, 60-63.

(59) Morikawa, T.; Matsuda, H.; Nishida, N.; Ohgushi, T.; Yoshikawa, M., Structures of new aromatics glycosides from a Japanese folk medicine, the roots of Angelica furcijuga. Chem. Pharm. Bull. 2004, 52, 1387-1390.

(60) Ren, Y.; Lantvit, D. D.; de Blanco, E. J. C.; Kardono, L. B. S.; Riswan, S.; Chai, H.; Cottrell, C. E.; Farnsworth, N. R.; Swanson, S. M.; Ding, Y.; Li, X. C.; Marais, J. P. J.; Ferreira, D.; Kinghorn, A. D., Proteasome-inhibitory and cytotoxic constituents of Garcinia lateriflora: absolute configuration of caged xanthones. Tetrahedron 2010, 66, 5311-5320.

(61) Cao, S. G.; Sng, V. H. L.; Wu, X. H.; Sim, K. Y.; Tan, B. H. K.; Pereira, J. T.; Goh, S. H., Novel cytotoxic polyprenylated xanthonoids from Garcinia gaudichaudii (Guttiferae). Tetrahedron 1998, 54, 10915-10924. 
(62) Arisawa, M.; Fujita, A.; Hayashi, T.; Morita, N.; Kikuchi, T.; Tezuka, Y., Studies on cytotoxic constituents in pericarps of Mallotus japonicus. IV. Chem. Pharm. Bull. 1990, 38, 698-700.

(63) Wang, Y.; Curtis-Long, M. J.; Lee, B. W.; Yuk, H. J.; Kim, D. W.; Tan, X. F.; Park, K. H., Inhibition of tyrosinase activity by polyphenol compounds from Flemingia philippinensis roots. Bioorg. Med. Chem. 2014, 22, 1115-1120.

(64) Ali, S.; Goundar, R.; Sotheeswaran, S.; Beaulieu, C.; Spino, C., Benzophenones of Garcinia pseudoguttifera (Clusiaceae). Phytochemistry 2000, 53, 281-284.

(65) Radwan, M. M.; ElSohly, M. A.; Slade, D.; Ahmed, S. A.; Khan, I. A.; Ross, S. A., Biologically active cannabinoids from high-potency Cannabis sativa. J. Nat. Prod. 2009, 72, 906-911.

(66) Rukachaisirikul, V.; Naklue, W.; Phongpaichit, S.; Towatana, N. H.; Maneenoon, K., Phloroglucinols, depsidones and xanthones from the twigs of Garcinia parvifolia. Tetrahedron 2006, 62, 8578-8585.

(67) Ueda, K.; Tsujimori, M.; Kodani, S.; Chiba, A.; Kubo, M.; Masuno, K.; Sekiya, A.; Nagai, K.; Kawagishi, H., An endoplasmic reticulum (ER) stress-suppressive compound and its analogues from the mushroom Hericium erinaceum. Bioorg. Med. Chem. 2008, 16, $9467-9470$.

(68) Xu, J.; Sun, X.; Liu, X.; Peng, M.; Li, S.; Jin, D.-Q.; Lee, D.; Bartlam, M.; Guo, Y., Phytochemical constituents from Melicope pteleifolia that promote neurite outgrowth in PC12 cells. J. Funct. Food. 2016, 23, 565-572.

(69) Popoola, O. K.; Marnewick, J. L.; Rautenbach, F.; Iwuoha, E. I.; Hussein, A. A., Acylphloroglucinol derivatives from the South African Heliehrysum niveum and their biological activities. Molecules 2015, 20, 17309-17324.

(70) Li, Y. C.; Kuo, Y. H., Two new isoflavones from the bark of Ficus microcarpa. J. Nat. Prod. 1997, 60, $292-293$. 
(71) Han, C.; Shao, T.; Song, X.; Chen, G.; Li, X. New isoflavone compound ficaurisoflavone B (3-(3-(2,3-dihydroxy-3-methylbutyl)4-hydroxyphenyl)-5,7-dihydroxy-4H-chromen-4-one) in Ficus auriculata, and its preparation method and application in inhibiting tumor cell. CN108314669A, 2018.

(72) Tanaka, H.; Etoh, H.; Shimizu, H.; Makita, T.; Tateishi, Y., Two new isoflavonoids from Erythrina variegata. Planta Med. 2000, 66, 578579.

(73) Ali, M. S.; Ali, M. I.; Ahmed, Z.; Onocha, P. A., Two new prenylflavanones from Erythrina sigmoidea. Nat. Prod. Commun. 2011, 6, 10991102.

(74) Wang, B. T.; Qi, Q. Y.; Ma, K.; Pei, Y. F.; Han, J. J.; Xu, W.; Li, E. W.; Liu, H. W., Depside $\alpha$-glucosidase inhibitors from a culture of the mushroom Stereum hirsutum. Planta Med. 2014, 80, 918-924. 